\title{
Synthesizing Linked Data Under Cardinality and Integrity Constraints
}

\author{
Amir Gilad ${ }^{*}$ \\ Duke University \\ agilad@cs.duke.edu
}

\author{
Shweta Patwa* \\ Duke University \\ sjpatwa@cs.duke.edu
}

\author{
Ashwin Machanavajjhala \\ Duke University \\ ashwin@cs.duke.edu
}

\begin{abstract}
The generation of synthetic data is useful in multiple aspects, from testing applications to benchmarking to privacy preservation. Generating the links between relations, subject to cardinality constraints (CCs) and integrity constraints (ICs) is an important aspect of this problem. Given instances of two relations, where one has a foreign key dependence on the other and is missing its foreign key $(F K)$ values, and two types of constraints: (1) CCs that apply to the join view and (2) ICs that apply to the table with missing $F K$ values, our goal is to impute the missing $F K$ values such that the constraints are satisfied. We provide a novel framework for the problem based on declarative CCs and ICs. We further show that the problem is NP-hard and propose a novel two-phase solution that guarantees the satisfaction of the ICs. Phase I yields an intermediate solution accounting for the CCs alone, and relies on a hybrid approach based on CC types. For one type, the problem is modeled as an Integer Linear Program. For the others, we describe an efficient and accurate solution. We then combine the two solutions. Phase II augments this solution by incorporating the ICs and uses a coloring of the conflict hypergraph to infer the values of the $F K$ column Our extensive experimental study shows that our solution scales well when the data and number of constraints increases. We further show that our solution maintains low error rates for the CCs.
\end{abstract}

\section{ACM Reference Format:}

Amir Gilad, Shweta Patwa, and Ashwin Machanavajjhala. 2021. Synthesizing Linked Data Under Cardinality and Integrity Constraints. In Proceedings of the 2021 International Conference on Management of Data (SIGMOD '21), fune 20-25, 2021, Virtual Event, China. ACM, New York, NY, USA, 13 pages. https://doi.org/10.1145/3448016.3457242

\section{INTRODUCTION}

In recent years, we have witnessed an increase in data-centric applications that call for efficient testing over reliable databases with certain desired qualities [11, 32]. Existing benchmarks such as TPC$\mathrm{H}[41,52]$ may not possess the desired characteristics for testing a specific application as they may not have the needed statistical qualities or the correct Integrity Constraints (ICs). The field of data generation $[5,9,19,22,25,35,42,44]$ has proven effective in this

${ }^{*}$ Both authors contributed equally to this research.

Permission to make digital or hard copies of all or part of this work for personal or classroom use is granted without fee provided that copies are not made or distributed for profit or commercial advantage and that copies bear this notice and the full citation on the first page. Copyrights for components of this work owned by others than ACM must be honored. Abstracting with credit is permitted. To copy otherwise, or republish, to post on servers or to redistribute to lists, requires prior specific permission and/or a fee. Request permissions from permissions@acm.org.

SIGMOD '21, fune 20-25, 2021, Virtual Event, China

(C) 2021 Association for Computing Machinery.

ACM ISBN 978-1-4503-8343-1/21/06 . \$ \$15.00

https://doi.org/10.1145/3448016.3457242 respect. Two prominent challenges in this field are: (1) the generation of links between different tables, i.e., aligning foreign keys with primary keys based on Cardinality Constraints (CCs) [5], and (2) ensuring that the data will satisfy a set of expected ICs [48].

In particular, when the real data is sensitive and access to it is heavily regulated, users often need to wait months or years to get access to the real data before they can even start writing data analysis programs. One solution is to generate realistic synthetic data that satisfies some CCs and ICs so that users can: (a) start writing code to analyse the data, (b) test it locally, and (c) evaluate whether access to the data would be useful for their purposes even before they get access to the real data. However, current methods for generating synthetic data under privacy constraints (especially state-of-the-art standards like differential privacy [17]) do not handle data with a combination of CCs or statistical constraints and ICs. Most, (e.g., [24, 47, 56]), only handle statistical constraints.

Furthermore, there has been a lot of recent work on answering count queries under differential privacy (e.g., Matrix mechanism [30], HDMM [36]) and in particular over relational databases [28]. A key challenge when answering queries especially over relational databases is that of consistency - are the answers outputted by a differentially private algorithm consistent with some underlying database? While there is work on using inference to enforce consistency when all the count queries are over a single view of the underlying database [23], these techniques do not extend to the case when: (a) the underlying database is relational and query answers are over several joined views of the relations, and (b) when the underlying database needs to satisfy some ICs. One solution to this problem is to find a database that is consistent with the query answers and the ICs, and answer queries from it. While techniques for finding such a consistent database are known for single tables without ICs [7, 23, 29], no such techniques are known when there are multiple tables in a relational database with ICs.

Moreover, DBMS testing and other applications may require databases that conform to both CCs and ICs to make them more realistic [5, 48]. For instance, consider a table with the attributes $A$ and $B$. A query grouping over attributes $A$ and $B$ could return as many tuples as the cross product of the active domains of $A$ and $B$. However, if there is a Functional Dependency $A \rightarrow B$, then the output size of the group-by query is only the maximum of the active domains of the two attributes. Thus, the presence of ICs can significantly impact the performance characteristics of queries.

In this paper, we investigate the problem of generating the links between database tables based on a set of linear CCs and a set of ICs.

Formally, we consider two relations, $R_{1}$ and $R_{2}$, where $R_{1}$ has a foreign key dependence on $R_{2}$ and is missing all values in its foreign key column $F K$. The goal is to impute $F K$ in $R_{1}$ based on the given CCs and ICs. Importantly, this problem and our solutions can be 
Persons (rel. $R_{1}$ )

\begin{tabular}{|c|c|c|c||c|}
\hline$p_{\text {id }}$ & Age & Rel & Multi-ling & $h_{\text {id }}$ \\
\hline 1 & 75 & Owner & 0 & $?$ \\
\hline 2 & 75 & Owner & 1 & $?$ \\
\hline 3 & 25 & Owner & 0 & $?$ \\
\hline 4 & 25 & Owner & 1 & $?$ \\
\hline 5 & 24 & Spouse & 0 & $?$ \\
\hline 6 & 10 & Child & 1 & $?$ \\
\hline 7 & 10 & Child & 1 & $?$ \\
\hline 8 & 30 & Owner & 0 & $?$ \\
\hline 9 & 30 & Owner & 1 & $?$ \\
\hline
\end{tabular}

Housing (rel. $R_{2}$ )

\begin{tabular}{|c|c|}
\hline$h_{i d}$ & Area \\
\hline 1 & Chicago \\
\hline 2 & Chicago \\
\hline 3 & Chicago \\
\hline 4 & Chicago \\
\hline 5 & NYC \\
\hline 6 & NYC \\
\hline
\end{tabular}

Figure 1: Database $\mathcal{D}$ with FK $h_{i d}$ missing from $R_{1}$



(a) Denial Constraints: $D C_{O, O}$ enforces that no two homeowners can reside in the same home, $D C_{O, S, l o w}$ and $D C_{O, S, u p}$ together specify the permissible age range of a spouse in any home, and $D C_{O, C, \text { low }}$ and $D C_{O, C, u p}$ give the age range for a child living with a multi-lingual homeowner

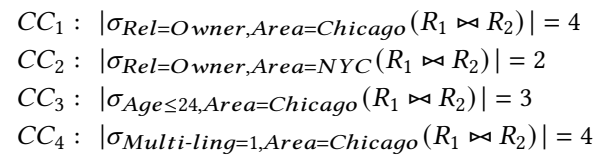

(b) Cardinality Constraints: $C C_{1}$ and $C C_{2}$ give the number of homeowners in Chicago and $\mathrm{NYC}$, resp., $C C_{3}$ gives the number of people younger than 25 who live in Chicago, and $C C_{4}$ gives the number of multi-lingual individuals in Chicago. Figure 2: Set of DCs and set of CCs

extended to relational databases with a snowflake schema [13], by focusing on pairs of relations linked by foreign key joins.

EXAMPLE 1.1. Consider the relations in Figure 1 based on the Census database. $R_{1}$ describes people through attributes such as age, relationship to a household (e.g. owner or spouse), whether they speak more than 1 language and a (missing) household id, whereas $R_{2}$ shows the area for each household. In addition, we are given the set of ICs and CCs in Figures $2 a$ and $2 b$, respectively. The goal is to impute values in the $h_{i d}$ column in $R_{1}$ so that the ICs and CCs are satisfied.

We believe that the problem we focus on is a key building block for the general problem of synthesizing data consistent with CCs and ICs for all three use-cases mentioned above. In particular, we believe that one can use the wealth of existing literature to synthesize individual relations consistent with CCs without the key relationships and then use our technique to fill-in the foreign keys.

\section{Our Contributions}

We model the problem, give a theoretical analysis, and provide a solution for the generation of foreign keys for existing database relations while ensuring the satisfaction of a set of ICs and reducing the error of a set of CCs. Next, we give our main contributions.
Model and Theoretical Results: We define the problem of CExtension whose input is a relation $R_{1}$ with an unknown foreign key dependence on a relation $R_{2}$, i.e., the $F K$ column in $R_{1}$ is missing, and a set of CCs and ICs. For the CCs, we define and use linear CCs that apply to $R_{1} \bowtie R_{2}$, based on [5]. For the ICs, we define a type of Denial Constraints (DCs) $[14,16]$, called Foreign Key DCs, that applies to $R_{1}$ and forbids tuples from having the same $F K$ value under specified conditions. We then show that $C$-Extension is NP-hard in data complexity. This result leads us to a two-phase heuristic solution that still ensures the satisfaction of all DCs, while tolerating possible errors in the CC counts.

Solution: Our solution can be split into two phases: (1) first phase (Section 4) is designed for the completion of a view $V_{J o i n}$ based on CCs, where $V_{J o i n}$ represents $R_{1} \bowtie R_{2}$ and is initialized with a copy of $R_{1}$ (without the $F K$ column) along with an empty column per nonkey column in $R_{2}$ (due to foreign key dependence, $\left|R_{1}\right|=\left|V_{\text {Join }}\right|$ ), and (2) second phase (Section 5) uses the generated view $V_{J o i n}$ to complete the $F K$ column in $R_{1}$ so that the DCs are satisfied.

Phase I: We provide a novel description of CC relationships that allows for $V_{\text {Join }}$ to be completed efficiently and precisely under specific conditions (presented in Section 3.1). We further devise algorithms for this case and the general case:

- For the general case, we devise an algorithm that models the CCs and the tuples in $V_{J o i n}$ as an Integer Linear Program (inspired by [5]). From its solution, we greedily infer the values in $V_{J o i n}$ for the attributes that come from $R_{2}$.

- For the special case, we devise a novel algorithm based on relationships between the CCs. We show that if the CCs have containment or disjointness relationships between them (defined in Section 4.2), then we can find an exact completion of $V_{J o i n}$ without any errors, provided one exists.

Our approach is a hybrid of these two solutions that employs the first solution for the subset of CCs that does not fit the special case, and employs the second solution for the subset of CCs that does.

Another novelty in our solution exploits the fact that the all-way marginals for $R_{1}$, i.e., counts of tuples with different combinations of values in $R_{1}$ 's non-key columns, have the same counts in $V_{\text {Join }}$. Thus, we augment the input set of CCs to improve accuracy.

Phase II: For the second phase, we employ the concept of a conflict hypergraph [16] and use a novel algorithm based on hypergraph coloring. We model the tuples in $R_{1}$ as vertices and connect by an edge every set of tuples that will violate a DC if assigned the same foreign key. Thus, colors represent the values that the foreign keys can take in $R_{1}$, and a proper coloring represents a mapping of tuples to foreign keys that does not violate any DC. Due to the previous stage that considered $R_{1} \bowtie R_{2}$, tuples in $R_{1}$ have a certain list of permitted colors. This version of the graph coloring problem is called List Coloring [2] and is known to be NP-hard. To color the graph, we use a greedy coloring algorithm that considers vertices in descending order by degrees. The algorithm skips vertices whose list of permitted colors is subsumed by the colors assigned to their neighbors. We ensure a proper coloring by adding the least number of new colors for the skipped vertices. Adding colors beyond the permitted lists corresponds to artificially adding tuples in $R_{2}$.

Experimental Evaluation We have implemented our solution and performed a comprehensive set of experiments on a dataset derived from the 2010 U.S. Decennial Census [45]. We have evaluated 
our solution in terms of accuracy and scalability in various scenarios, several of which were used for comparison with a baseline based on [5]. We further examined the runtime breakdown of our approach, presenting the runtimes of phases I and II in our solution. Our results indicate that our solution incurs relatively small error for CCs and no error for DCs (as guaranteed by our theoretical analysis). Moreover, our algorithms scale well for large data sizes, and large and complex sets of CCs and DCs. For increasing data scales, our approach was 17 times faster on average across different cases than the baseline we compare to.

\section{PRELIMINARIES AND MODEL}

We now define the basic concepts used throughout the paper, and the C-Extension problem.

Relations in a Database: Let $R_{1}$ and $R_{2}$ be relations over the schema attributes $\left(K_{1}, A_{1}, \ldots, A_{p}, F K\right)$ and $\left(K_{2}, B_{1}, \ldots, B_{q}\right)$, respectively. An attribute $A_{j}$ of $R_{i}$ may also be called a column and is denoted by $R_{i} . A_{j} . t \in R_{i}$ denotes a tuple in $R_{i}$ and $t . A_{j}$ denotes the cell of column $A_{j}$ in tuple $t$. The last column in $R_{1}(F K)$ is a foreign key column that gets its values from the key column $K_{2}$ in $R_{2}$. The view $V_{\text {Join }}=R_{1} \bowtie_{F K=K_{2}} R_{2}$ denotes the join of the two relations. If all values of a column $A_{i}$ are missing, it is called a missing column.

EXAMPLE 2.1. Consider a database $\mathcal{D}$ with two relations $R_{1}$ and $R_{2}$ as shown in Figure 1. $R_{1} . h_{i d}$ is a missing column. The first row in $R_{1}$ says that $t_{1}$.Age is $75, t_{1}$.Rel is Owner and $t_{1}$.Multi-ling is 0 .

Foreign Key Denial Constraints: DCs [14] are a general form of constraints that can be written as a negated First Order Logic statement. DCs can express several types of integrity constraints like functional dependencies and conditional functional dependencies [10]. In this paper, we restrict our attention to DCs that contain a condition of the form $t_{1} \cdot F K=\ldots=t_{k} . F K$.

Definition 2.2 (Foreign Key DC). A Foreign Key DC on a relation $R\left(K_{1}, A_{1}, \ldots, A_{p}, F K\right)$ is defined as the following FOL statement:

$$
\forall t_{1}, t_{2}, \ldots, t_{k} . \quad \neg\left(p_{1} \wedge \ldots \wedge p_{n}\right)
$$

where $p_{q}=t_{i} \cdot A_{l} \circ t_{j} \cdot A_{l}$ or $p_{q}=t_{i} \cdot A_{l} \circ c$, for $t_{i}, t_{j} \in R, p \geq 2$, ○ $\in$ $\{=,<,>, \neq\}, c$ and $k$ are constants, and $p_{n}=\left(t_{1} \cdot F K=\ldots=t_{k} \cdot F K\right)$.

We use the terms Foreign Key DC and DC interchangeably.

EXAMPle 2.3. DC $O, O$ (Figure 2a), which states that two homeowners cannot be in the same home, can be formulated as follows:

$$
\forall t_{1}, t_{2} \in R_{1} . \neg\left(t_{1} \cdot \text { Rel }=t_{2} \cdot \text { Rel }=\text { O wner } \wedge t_{1} \cdot h_{i d}=t_{2} \cdot h_{i d}\right)
$$

Note that the restriction to Foreign Key DCs means that all constraints are on people that are in the same household.

Linear Cardinality Constraints: CCs form the second class of constraints that allows for the specification of the number of tuples that should posses a certain set of attribute values, which can be expressed as a selection condition. As standard in previous work $[5,37]$, we restrict our attention to linear CCs.

Definition 2.4 (LineAR CC, AdAPted FRom [5]). A linear CC over a database $\mathcal{D}$ consisting of relations $R_{1}\left(K_{1}, A_{1}, \ldots, A_{p}, F K\right)$ and $R_{2}\left(K_{2}, B_{1}, \ldots, B_{q}\right)$ is defined as follows:

$$
\left|\sigma_{\varphi}\left(R_{1} \bowtie_{F K=K_{2}} R_{2}\right)\right|=k
$$

where $\varphi$ is a Boolean selection predicate over a subset of (non-key) attributes in $\mathcal{D}$, and $k \in \mathbb{N}$.
In the rest of the paper, we only refer to conjunctive selection predicates with conjuncts of the form $A_{i} \circ c$, where $\circ \in\{=,<,>, \leq$ $, \geq\}$ and $c$ is in the domain of column $A_{i}$, though our algorithms can be extended to conditions that contain disjunction as well.

EXAMPLE 2.5. $C C_{1}$ (Figure 2b), which states that the number of homeowners (Rel $=$ Owner) living in Area $=$ Chicago must equal 4, can be written as: $\left|\sigma_{\text {Rel }=O \text { wner,Area }=\text { Chicago }} R_{1} \bowtie R_{2}\right|=4$.

We denote by $R \vDash \sigma$ the fact that relation $R$ meets constraint $\sigma$. Problem Definition: We now formally define the C-Extension problem and discuss its intractability.

Definition 2.6 (C-Extension). Let $R_{1}\left(K_{1}, A_{1}, \ldots, A_{p}, F K\right)$ and $R_{2}\left(K_{2}, B_{1}, \ldots, B_{q}\right)$ be two relations, where $R_{1} . F K$ is a foreign key mapped from $R_{2} . K_{2}$ and is empty. Let $S_{D C}$ denote the set of DCs over $R_{1}$ and let $S_{C C}$ denote the set of linear CCs over the foreign key join between $R_{1}$ and $R_{2}$. C-Extension is the problem of completing all the values in $R_{1} . F K$ to create $\hat{R}_{1}$ so that (1) $\forall \sigma \in S_{D C}, \hat{R_{1}} \vDash \sigma$, (2) $\forall \sigma \in S_{C C}, \hat{R_{1}} \triangleleft_{F K=K_{2}} R_{2} \vDash \sigma$.

EXAMPLE 2.7. Reconsider relations $R_{1}$ and $R_{2}$ in Figure 1, and DCs and CCs in Figure 2. A solution $\hat{R_{1}}$ for the $C$-Extension problem as defined by these relations and constraints is shown in Figure 3.

\begin{tabular}{|c|c|c|c||c|}
\multicolumn{7}{|c|}{ Persons (rel. $R_{1}$ ) } \\
\hline$p_{\text {id }}$ & Age & Rel & Multi-ling & $h_{\text {id }}$ \\
\hline 1 & 75 & Owner & 0 & 2 \\
\hline 2 & 75 & Owner & 1 & 1 \\
\hline 3 & 25 & Owner & 0 & 3 \\
\hline 4 & 25 & Owner & 1 & 4 \\
\hline 5 & 24 & Spouse & 0 & 2 \\
\hline 6 & 10 & Child & 1 & 2 \\
\hline 7 & 10 & Child & 1 & 2 \\
\hline 8 & 30 & Owner & 0 & 5 \\
\hline 9 & 30 & Owner & 1 & 6 \\
\hline
\end{tabular}

Figure 3: Relation $R_{1}$ from Figure 1 with FK $h_{i d}$ filled-in to satisfy DCs and CCs given in Figure 2

The decision version of C-Extension is given by the same setting as in Definition 2.6. The output is 1 if there exists a completion of $R_{1} . F K$ such that all DCs and CCs are satisfied, and 0 otherwise.

Proposition 2.8. The decision problem version of C-Extension is NP-hard in data complexity.

Proof Sketch. We describe a reduction from NAE-3SAT to CExtension. In the NAE-3SAT problem, we are given a 3-CNF formula $\varphi$ and asked whether there is a satisfying assignment to $\varphi$ with every clause having at least one literal with the value False. Given a 3-CNF formula $\varphi=C_{1} \wedge \ldots \wedge C_{n}$, where $x_{1}, \ldots, x_{m}$ are the propositional variables in $\varphi$, construct a relation $R_{1}(\operatorname{Var}, \alpha, C l s, C h o s e n)$, where Chosen is missing all values, and $\operatorname{Var}, \alpha, C l s$ columns take values:

(1) $\left(x_{i}, 1, C_{j}\right.$, ?) if making $x_{i}$ True makes $C_{j}$ True

(2) $\left(x_{i}, 0, C_{j}\right.$, ?) if making $x_{i}$ False makes $C_{j}$ True

We define $S_{D C}$ to be the set with the following two DCs:

(1) $\forall t_{1}, t_{2} . \neg\left(t_{1} . V a r=t_{2} . V\right.$ ar $\wedge t_{1} . \alpha \neq t_{2} . \alpha \wedge t_{1}$.Chosen $=t_{2}$.Chosen $)$

(2) $\forall t_{1}, t_{2}, t_{3} . \neg\left(t_{1} . C l s=t_{2} . C l s=t_{3} . C l s \wedge t_{1}\right.$.Chosen $=t_{2}$.Chosen $=t_{3}$.Chosen $)$

CCs are not needed in the reduction. The goal is to complete the missing column Chosen in $R_{1}$. We define $R_{2}$ as containing two columns: a primary key column Chosen, and another column E. $R_{2}$ contains the tuples $(0, a)$ and $(1, b)$, i.e., the domain for Chosen is 
$\{0,1\}$. Intuitively, Chosen encodes the satisfying assignment for $\varphi$ by assigning values to each tuple, where $t$.Chosen $=1$ iff the assignment should be $t . V a r=t . \alpha$.

The full proofs are detailed in the full version [21].

\section{SOLUTION OVERVIEW}

Our solution proceeds in two phases as seen in Figure 4. In phase I, we consider the view $V_{\text {Join }}$ representing the join of the two relations $R_{1}$ and $R_{2}$, where $R_{1}$ has a foreign key dependence on $R_{2}$, and initialize it with (non $F K$ ) columns from $R_{1}$ and an empty column per non-key column from $R_{2}$. We infer these values based on the CCs by a hybrid approach that uses both ILP [5] and a more efficient and accurate procedure for special cases. In phase II, we impute $R_{1}$.FK by modeling the problem as a conflict hypergraph using the DCs, and coloring it based on the inferred values in $V_{J o i n}$.

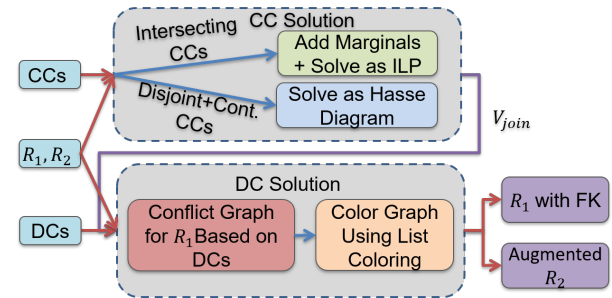

Figure 4: Solution Overview

\subsection{Overview of the First Phase}

Due to the foreign key dependence (Definition 2.6), we define $V_{\text {Join }}$ over the columns $K_{1}, A_{1}, \ldots, A_{p}, B_{1}, \ldots, B_{q}$ such that $t \in R_{1}$ implies that there is a single $t^{\prime} \in V_{\text {Join }}$ with $t . K_{1}=t^{\prime} . K_{1}$ and $\forall 1 \leq i \leq$ p. $t . A_{i}=t^{\prime} . A_{i}$ with additional $B_{1}, \ldots, B_{q}$ entries that are initially all empty because $F K$ is missing in $R_{1}$. Therefore, $\left|V_{\text {Join }}\right|=\left|R_{1}\right|$. Our goal is to complete these columns based on the CCs.

EXAmple 3.1. Reconsider $R_{1}$ and $R_{2}$ shown in Figure 1 and the $C C s$ in Figure $2 b$. The join view $V_{\text {Join }}$ is $R_{1}$ as it appears in Figure 1 (without $h_{i d}$ ) with an empty Area column (as this is the schema of $\left.R_{1} \bowtie R_{2}\right)$. Due to the foreign key dependency, we have $\left|V_{J o i n}\right|=\left|R_{1}\right|$, and $V_{\text {Join }}$ contains a tuple for each $R_{1}$ tuple with the same values as in $R_{1}$ and an empty Area value. The reason is that the $F K$ values are missing in $R_{1}$. Our goal is to fill-in $V_{J o i n}$ so that the CCs are satisfied.

We give a short description of our solution for completing $V_{\text {Join }}$ Solution as an ILP (Section 4.1, green box in Figure 4): Given a set of CCs on $V_{J o i n}$, we model the problem of completing the missing columns as a system of linear equations with variables accounting for counts of different tuples needed in $V_{\text {Join }}$ to satisfy the CCs. Thus, the variables must take non-negative integer values. We artificially add to $S_{C C}$ all-way marginals (using the idea of intervalization from [5] that is explained in Section 4) from $R_{1}$ to enhance the accuracy of the solution. For example, based on CCs given in Example 1.1, $\left|\sigma_{\text {Age } \leq 24, \text { Rel=Spouse,Multi-ling=0 }}\right|=1$ gets added to $S_{C C}$. We then assign $B_{1}, \ldots, B_{q}$ values to the tuples in $V_{\text {Join }}$ based on the solution returned by an ILP solver.

Using CC Relationships for Special Cases (Section 4.2, blue box in Figure 4): We give a novel description of the relationships between CCs based on their selection conditions, defining CC containment, disjointness and intersection. In the case where there are no intersecting CCs and no disjunctions, we give an algorithm to complete $V_{\text {Join }}$ that models the containment and disjointness of CCs as a Hasse diagram [53] that it recurses on bottom-up to fill-in $V_{\text {Join }}$. Any leftover $V_{\text {Join }}$ tuples without $B_{1}, \ldots, B_{q}$ values are randomly assigned a combination that cannot cause a new contribution towards the target count of any CC. However, if no such combinations are available, then the leftover $V_{\text {Join }}$ tuples cannot be completed. We refer to these as invalid tuples.

Hybrid Approach (Section 4.3): In the absence of intersecting CCs, the solution decomposes cleanly as seen above. This motivates the hybrid approach that combines ideas from both cases to achieve better runtime and accuracy when some CCs intersect. We start by labeling each pair of CCs as disjoint, contained or intersecting. For all CCs that do not intersect or contain any intersecting CCs, we use the approach from Section 4.2, and for the rest, we use the ILP approach from Section 4.1. Lastly, as seen above in the special case, we may end up with some invalid tuples.

\subsection{Overview of the Second Phase}

After filling-in the columns of $V_{\text {Join }}$ that originate in $R_{2}\left(B_{1} \ldots, B_{q}\right)$, we turn to reverse-engineering $R_{1}$ from $V_{\text {Join }}$. This phase uses conflict hypergraphs [16] to represent possible DC violations.

Conflict Hypergraph (Section 5.1, red box in Figure 4): We use the notion of conflict hypergraph for the tuples of $R_{1}$ based on the DCs. Given a DC, we construct an edge for all the sets of tuples that cannot get the same foreign key value due to that DC.

EXAMPLE 3.2. Consider the relation $R_{1}$ depicted in Figure 1 and the first DC in Figure 2a. Suppose the first two tuples are assigned the same Area value in $V_{J o i n}$. Thus, the conflict hypergraph will have an edge containing the tuples with $p_{i d}=1$ and $p_{i d}=2$ since they are both owners and cannot be in the same household (the $h_{i d}$ value). The conflict hypergraph of our running example is depicted in Figure 7.

List Coloring (Section 5.1, orange box in Figure 4): Proper coloring of the hypergraph ensures that there must be at least two vertices in each edge with distinct colors. Thus, modeling each FK value as a color and each tuple as a vertex allows us to prove that a proper coloring results in an assignment of $F K$ values that satisfies the DCs. The values in $V_{\text {Join }}$ filled-in by the previous phase induce a list of possible $F K$ values, and thus colors, for $R_{1}$ tuples. Finding a proper coloring such that each vertex assumes a color from its predefined list is called List Coloring [2] and is NP-hard. We thus propose a greedy coloring algorithm based on vertex degree.

Algorithm for Satisfying the DCs (Section 5.2): The size of the conflict hypergraph can be very large and thus may cause a significant slowdown in practice. Therefore, we partition $R_{1}$ into smaller sets with the same $B_{1} \ldots, B_{q}$ values and construct a conflict hypergraph for each set separately. For each non-invalid tuple, $V_{J o i n}$ contains $B_{1} \ldots, B_{q}$ values, so we can use our greedy coloring algorithm to find a coloring for them. We color invalid tuples at the end using all $F K$ values as candidates. This phase may result in the addition of extra tuples to $R_{2}$ (the second output in Figure 4).

\section{FIRST PHASE: SOLVING CCS}

In this section, we focus on the first phase. Given two relations $R_{1}\left(K_{1}, A_{1}, \ldots, A_{p}, F K\right)$ and $R_{2}\left(K_{2}, B_{1}, \ldots, B_{q}\right)$, we wish to satisfy a set $S_{C C}$ of CCs over the join view $V_{\text {Join }}=R_{1} \bowtie_{F K=K_{2}} R_{2}$. 


\subsection{Solution as an ILP}

We give a two-part solution in Algorithm 1 where we: (1) model the CCs as a system of linear equations and solve it using an ILP solver, and (2) greedily fill-in $B_{1}, \ldots, B_{q}$ values for each tuple in $V_{\text {Join. }}$. The first part (lines 3-15) is inspired by [5]. Each variable represents the number of tuples with a specific combination of $A_{1}, \ldots, A_{p}, B_{1}, \ldots, B_{q}$ values in $V_{\text {Join }}$. Each CC is written as a sum of the variables whose associated tuples satisfy its selection condition. We now introduce the notion of intervalization [5].

Intervalization: Creating a variable for every combination of values in the cross product of the full domains of all the $p+q$ (non-key) columns in $V_{\text {Join }}$ would give a very large ILP. We augment the notion of intervalization [5] so that it will not only assist in reducing the number of variables based on the intervals of values in $S_{C C}$, but also use only the combinations of $A_{1}, \ldots, A_{p}$ values already in $R_{1}$. We call this binning the distinct $\left(A_{1}, \ldots, A_{p}\right)$ values in $R_{1}$.

In the system of equations $A x=b$, row $r_{i}$ (in $A$ ) corresponds to $C C_{i}$ and row $b_{i}$ (in $b$ ) stores $C C_{i}$ 's target count. We create the vector $x$ of variables by putting bins with the same $B_{1}, \ldots, B_{q}$ values as contiguous elements (see Example 4.1). Since input CCs are linear, each element in $A$ is 0 or 1 . The goal is to solve for an $x$ with nonnegative integer entries (line 15). Such a solution can be obtained if there exists a solution to C-Extension where $R_{1} \bowtie_{F K=K_{2}} R_{2}$ satisfies $S_{C C}$. In the second part (lines 17-19), we fill-in the $B_{1}, \ldots, B_{q}$ values greedily. For each assignment $x_{i}=v_{i}$, we find at most $v_{i}$ tuples (with empty $B_{1}, \ldots, B_{q}$ cells) in $V_{\text {Join }}$ that satisfy $x_{i}$ 's selection condition on $R_{1}$, and fill-in their $B_{1}, \ldots, B_{q}$ values as encoded by $x_{i}$.

EXAMPLE 4.1. Reconsider relations $R_{1}$ and $R_{2}$ in Figure 1, CCs in Figure $2 b$ and $V_{\text {Join }}$ described in Example 3.1. Intervalization splits Age into [0,24] and [25,114] due to $\mathrm{CC}_{3}$ (all other columns are categorical). Even though $R_{1}$ contains multiple tuples for multi-lingual homeowners with age greater than 24, it suffices to look at those with Age in [0,24] and [25,114]. Importantly, for the given instance, we only need to keep track of the following tuple types: (1) Age $\in$ [25, 114], Rel = Owner, Multi-ling = 0, (2) Age $\in$ [0,24], Rel = Spouse, Multi-ling = 0, (3) Age $\in$ [0,24], Rel = Child, Multi-ling = 1 , and (4) Age $\in[25,114]$, Rel $=$ Owner, Multi-ling $=1$. Here, vector $x$ uses a copy of these four bins with Area $=$ Chicago in $x_{1}$ to $x_{4}$ and Area $=$ NYC in $x_{5}$ to $x_{8}$. Without the idea of binning, we would need 16 variables because Area can take 2 distinct values and $R_{1}$ contains 8 unique tuples. Finally, we iterate through each $C C_{i} \in S_{C C}$ and add rows $r_{i}$ and $b_{i}$ in $A$ and $b$, resp. For $C C_{1}, r_{i}=[1,0,0,1,0,0,0,0]$ and $b_{i}=4$ because only $x_{1}$ and $x_{4}$ match the selection conditions in $C C_{1}$; similarly for other CCs. Hence, $A x=b$ has a solution given by $x_{1}=2, x_{2}=1, x_{3}=2, x_{4}=2, x_{5}=1, x_{6}=0, x_{7}=0$ and $x_{8}=1$. Finally, we iterate through $x_{i}$ 's to find $V_{J o i n}$ tuples which satisfy its selection condition and assign the matching Area value that gives the view in Figure 5. E.g., we find two tuples in $V_{\text {Join }}$ with Age $\in[25,114]$, Rel $=$ Owner and Multi-ling $=0$ for $x_{1}$ and assign Area $=$ Chicago.

Augmenting with All-Way Marginals: When $A$ is sparse, some $x_{i}$ values in the solution may not match the true counts. Despite such discrepancies, we can complete several tuples in $V_{\text {Join }}$ because we update at most as many tuples as the value of $x_{i}$ in the solution. The order of updates may also impact which subset of $V_{\text {Join }}$ tuples gets specific $B_{1}, \ldots, B_{q}$ values. For example, another solution to the ILP in Example 4.1 is given by $x_{1}=0, x_{2}=3, x_{3}=0, x_{4}=4, x_{5}=$

\begin{tabular}{|c|c|c|c||c|}
\hline$p_{\text {id }}$ & Age & Rel & Multi-ling & Area \\
\hline 1 & 75 & Owner & 0 & Chicago \\
\hline 2 & 75 & Owner & 1 & Chicago \\
\hline 3 & 25 & Owner & 0 & Chicago \\
\hline 4 & 25 & Owner & 1 & Chicago \\
\hline 5 & 24 & Spouse & 0 & Chicago \\
\hline 6 & 10 & Child & 1 & Chicago \\
\hline 7 & 10 & Child & 1 & Chicago \\
\hline 8 & 30 & Owner & 0 & NYC \\
\hline 9 & 30 & Owner & 1 & NYC \\
\hline
\end{tabular}

Figure 5: Join view $V_{J o i n}=\left(R_{1} \bowtie_{F K=K_{2}} R_{2}\right)$ of $R_{1}$ and $R_{2}$ from Figure 1 with filled-in Area values



$x_{6}=x_{7}=0, x_{8}=2$. This assigns Area $=$ Chicago to tuples with $p_{i d}=2,4,5,9$ in $V_{\text {Join }}$. However, the remaining tuples do not get any Area value and no CC in $S_{C C}$ gets satisfied in $V_{J o i n}$. We overcome this issue by using both $S_{C C}$ and all all-way marginals over $A_{1}, \ldots, A_{p}$ from $R_{1}$ when solving the ILP (see the discussion about the baseline's CC accuracy in Section 6). The solution reported in Example 4.1 was computed with all all-way marginals.

Complexity: The complexity of Algorithm 1 is $O\left(\left|S_{C C}^{\prime}\right| \cdot m+S\right)$, where $S_{C C}^{\prime}$ contains CCs from $S_{C C}$ along with the marginals, and $m$ is the number of variables that is upper-bounded by the number of tuples in $R_{1}$ times the product of the sizes of the active domains of $B_{1}, \ldots, B_{q}$ in $R_{2}$. Lastly, $S$ is the time complexity of the ILP solver. 


\subsection{Efficient Algorithm for Special CC Types}

In practice, Algorithm 1 may incur slow runtimes as generating and solving the system of equations is time consuming, even with stateof-the-art ILP solvers (as shown in Section 6). Thus, we describe a model for relationships between the CCs in $S_{C C}$ and devise an algorithm to better tackle $V_{\text {Join }}$ completion in specific scenarios.

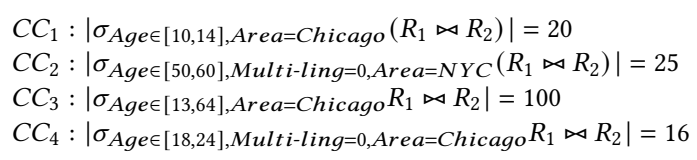

Figure 6: CC relationships. $C C_{1} \cap C C_{2}=\emptyset$, and $C C_{4} \subseteq C C_{3}$

Definition 4.2. $C C_{i}, C C_{j} \in S_{C C}$ are disjoint either if their selection conditions on the $R_{1}$ attributes are disjoint, or if their selection conditions on $R_{1}$ are identical and the conditions on $R_{2}$ are disjoint. We denote this by $C C_{i} \cap C C_{j}=\emptyset$.

Note that we also consider pairs of CCs with the same $R_{1}$, but disjoint $R_{2}$ selection conditions as disjoint. For a pair $\left(C C_{i}, C C_{j}\right)$ of such CCs, assigning $B_{1}, \ldots, B_{q}$ values in tuples that contribute to the count of $C C_{i}$ should not limit the set of tuples available for $C C_{j}$, if a solution exists. We label such pairs similarly to a pair of disjoint CCs. Next, we define the notion of CC containment.

Definition 4.3. Let $C C_{i}, C C_{j} \in S_{C C}$ such that $C C_{i}:\left|\sigma_{\varphi_{i}}(R)\right|=$ $k_{i}$ and $C C_{j}:\left|\sigma_{\varphi_{j}}(R)\right|=k_{j} . C C_{i}$ is contained in $C C_{j}$, denoted $C C_{i} \subseteq$ $C C_{j}$, if $\varphi_{i}$ uses a (non-strict) superset of attributes in $\varphi_{j}$ and for each common attribute, the values in $C_{i}$ are a subset of the corresponding values in $C C_{j}$.

Intuitively, if $C C_{i}$ is contained in $C C_{j}$, then $C C_{i}$ is more restrictive than $C C_{j}$, and assigning a tuple $t \in R_{1}$ values in $B_{1}, \ldots, B_{q}$ that satisfy the selection condition in $C C_{i}$ will also satisfy the selection condition in $C C_{j}$. This observation defines a partial order on $S_{C C}$ which we utilize later to find a solution for CCs.

Definition 4.4. $C C_{i}, C C_{j} \in S_{C C}$ are said to be intersecting if they are neither disjoint nor does one contain the other. We denote this by $C C_{i} \cap C C_{j} \neq \emptyset$.

EXAmple 4.5. Assume $R_{1}$ (or $V_{\text {Join }}$ ) contains 10 tuples with Age $\epsilon$ $[10,30), 20$ with Age $\in[30,50)$ and 50 with Age $\in[50,70]$. Let:

$$
\begin{aligned}
& C C_{1}:\left|\sigma_{\text {Age } \in[10,50), \text { Area }=\text { Chicago }} R_{1} \bowtie R_{2}\right|=30 \\
& C C_{2}: \mid \sigma_{\text {Age }} \in[30,70], \text { Area }=N Y C R_{1} \bowtie R_{2} \mid=30
\end{aligned}
$$

If all tuples with Age $\in[30,50)$ get assigned Area $=N Y C, C C_{1}$ cannot be satisfied. Even when Area $=$ Chicago in $C_{2}$, it is unclear how many tuples with age in $[30,50)$ can be assigned Area $=$ Chicago.

Solution Without Intersecting CCs: Now, we focus on the setting where there are no intersecting CCs present and describe Algorithm 2 that outputs an exact solution.

We use the notion of a Hasse diagram [53], denoted by $\mathcal{H}=$ $(V, E)$, to encode the containment relationships between the CCs in $S_{C C}$. We refer to each connected component in the undirected version of $\mathcal{H}$ as a diagram. Within each diagram, the $\mathrm{CC}$ that is not contained in any other $\mathrm{CC}$ is referred to as the maximal element.

Algorithm 2 is given the join view $V_{\text {Join }}$ with missing $B_{1}, \ldots, B_{q}$ columns, $S_{C C}$ and the Hasse diagram $\mathcal{H}$ describing the containment relations in $S_{C C}$. We denote by $\mathcal{V}(\mathcal{H})$ and $\mathcal{E}(\mathcal{H})$ the collective set of all nodes and edges of the diagrams in $\mathcal{H}$. The algorithm operates recursively with a single base case - if all the CCs in $S_{C C}$ are disjoint, i.e., $\mathcal{E}(\mathcal{H})$ is empty (line 2 ), then it simply chooses $k_{i}$ tuples that can contribute to each $C C_{i} \in S_{C C}$ and completes their $B_{1}, \ldots, B_{q}$ values given by $C C_{i}$. When the base case is not met, for each $H \in \mathcal{H}$, the algorithm makes a recursive call on each child of the maximal element $m$ in $H$ (lines 9-11) to get the resulting view of the subdiagram and then finds the remaining number of tuples that will get $C C_{m}$ to its target count (lines 12-13). Finally, in the loop in line 15 , the algorithm completes any missing values in the tuples while ensuring that these values do not add to the count of any $C C \in S_{C C}$ by finding combinations that are not specified in $S_{C C}$.

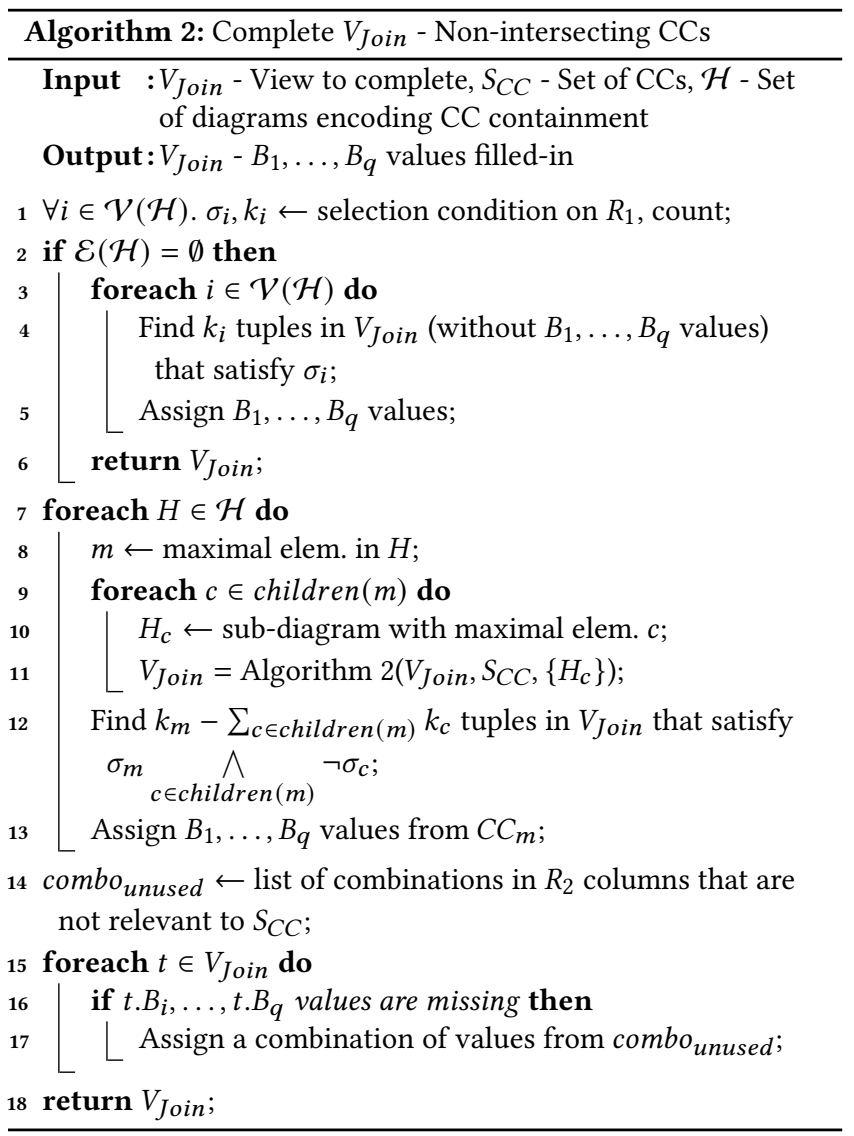

EXAmple 4.6. Reconsider CCs 1-4 in Figure 6. The set $\mathcal{H}$ is $\left\{H_{1}, H_{2}\right.$, $\left.\mathrm{H}_{3}\right\}$, where $\mathrm{H}_{1}$ and $\mathrm{H}_{2}$ contain only $\mathrm{CC}_{1}$ and $\mathrm{CC}_{2}$, respectively, and $\mathrm{H}_{3}$ is a diagram composed of one edge from $\mathrm{CC}_{3}$ to $\mathrm{CC}_{4}$. Algorithm 2 gets $\mathcal{H}$ along with $V_{\text {Join }}$ and $S_{C C}$ as input. It assigns $C C_{i}$ 's selection condition on $R_{1}$ and target count to $\sigma_{i}$ and $k_{i}$, for all $i$. Then, it checks the condition in line 2, which does not hold as we have the edge $\left(C C_{3}, C C_{4}\right)$. Thus, it goes to the loop in line 7 to iterate over the three diagrams. For $\mathrm{H}_{3}$, the maximal element is $\mathrm{CC}_{3}$, so Algorithm 2 recursively calls itself for the sub-diagram containing only $\mathrm{CC}_{4}$ (line 11) and finds 16 tuples such that Age $\in[18,24]$ and Multiling $=0$, and assigns to them Area $=$ Chicago (lines 3-5). It then returns from the recursive call to find $100-16=84$ tuples with Age $\in$ 
$[13,64] \backslash[18,24]$ and Multi-ling $\neq 0$, and assigns to them Area $=$ Chicago (lines 12-13). For $\mathrm{H}_{1}\left(\mathrm{H}_{2}\right)$ the maximal element is $\mathrm{CC}_{1}\left(\mathrm{CC}_{2}\right)$, the algorithm then performs a recursive call to itself with an empty diagram, and returns from the call to select 20 (25) tuples that have Age $\in[10,14]($ Age $\in[50,60]$ and Multi-ling =0) and assign to them Area $=$ Chicago $($ Area $=N Y C)$. Here, combo $_{\text {unused }}$ contains values from Area's domain except Chicago and NYC which get used in $S_{C C}$. If there are any tuples in $V_{\text {Join }}$ without an assignment (see loop on line 15), we assign to each a value chosen from combo $o_{\text {unused }}$.

At the end of the algorithm, any tuple in $V_{\text {Join }}$ without $B_{1}, \ldots, B_{q}$ values is randomly assigned a combination of values that is not used in $S_{C C}$ (line 17). We refer to these tuples as invalid tuples if no such combination is available. Observe that the matching tuples in $R_{1}$ do not have an $F K$ to $K_{2}$ mapping, i.e., if there is a tuple in $V_{\text {Join }}$ that is missing an assignment, also called an invalid tuple, then $V_{\text {Join }}$ does not give a set of candidate $K_{2}$ values that could be assigned in its $F K$ cell. We will handle such tuples in Section 5.2.

Proposition 4.7. If $S_{C C}$ does not contain intersecting $C C s$ and there exists a join view $V_{J o i n}$ that satisfies all CCs in $S_{C C}$, then Algorithm 2 finds such a view.

Complexity: The complexity of Algorithm 2 is $O\left(\left|S_{C C}\right|^{2} \cdot d_{1}+\right.$ $\left.\left|S_{C C}\right| \cdot\left(\max _{i}\left|\operatorname{dom}_{a}\left(B_{i}\right)\right|\right)^{d_{2}}+\left|S_{C C}\right| \cdot\left|V_{J o i n}\right|\right)$, where $d_{1}, d_{2}$ are the number of columns in $V_{\text {Join }}$ and $R_{2}$, $\operatorname{dom}_{a}\left(B_{i}\right)$ is the active domain of $R_{2} \cdot B_{i}$. The first term is for computing the relationships between CCs and recursing on the Hasse diagrams (lines 7-11), second term

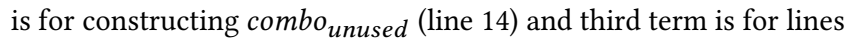
$1-6,12-13$ and choosing a random value per tuple in lines 15-17. In practice, we only consider columns used in $S_{C C}$ instead of $d_{2}$.

\subsection{Hybrid Approach}

In many cases, $S_{C C}$ contains a combination of disjoint, contained, and intersecting CCs, so we combine Algorithms 1 and 2.

We start by constructing Hasse diagram based on containment relationship between pairs of CCs in $S_{C C}$. Next, we iterate through each diagram $H \in \mathcal{H}$, and discard $H$ if it contains intersecting CCs. Note that the absence of an edge in the Hasse diagram does not guarantee the lack of intersection at the beginning of phase I (demonstrated by Example 4.5 where the Hasse diagram starts out as two nodes without an edge, but the CCs represented by these nodes do intersect). Therefore, we keep track of which CCs intersect to then discard the affected diagrams (set $S_{2}$ ) and run Algorithm 2 on the remaining diagrams (set $S_{1}$ ). In particular, $\forall C C_{i} \in S_{1}, C C_{j} \in$ $S_{2} . C C_{i} \cap C C_{j}=\emptyset, C C_{i} \nsubseteq C C_{j}$ and $C C_{j} \nsubseteq C C_{i}$. We then run Algorithm 2 for CCs in $S_{1}$, and Algorithm 1 for those in $S_{2}$.

As seen above, it is possible that some tuples may not have a $B_{1}, \ldots, B_{q}$ assignment in $V_{J o i n}$. Let $S_{3}$ be the set of these tuples that


$\mid$ combo $_{\text {unused }} \mid=\emptyset$, then all tuples in $S_{3}$ are invalid tuples.

Augmenting with Modified Marginals Our approach guarantees that the partial solution returned by Algorithm 2 satisfies $S_{1}$ exactly. In comparison to how we augment $S_{C C}$ with marginals in Section 4.1 before solving the ILP, we now want the scope of the marginals being added to be limited to the tuples that are relevant for the CCs in $S_{2}$. For example, let $S_{C C}=\left\{C C_{1}, C C_{3}\right\}$ from Figure $2 \mathrm{~b}$. We add CCs with the following selection predicates: (1) Age <=



Figure 7: Conflict graph for the tuples in $R_{1}$ from Figure 1 based on $V_{J o i n}$ from Figure 5. Two tuples are connected by a solid edge if their Area values match but they cannot be assigned the same $h_{i d}$ value. Dashed edges show DC violations between tuples with different Area values. Partitioning $V_{\text {Join }}$ by Area values addresses such violations because the set of household ids is disjoint for different Area values

24, Rel $=$ Owner, Multi-ling $=0$, and (2) Age $<=24$, Rel $=$ Owner, Multi-ling $=1$. It may still happen that the matrix $A$ is sparse and some $x_{i}$ 's do not match the true counts causing some CC errors.

\section{SECOND PHASE: ADDING DCS}

We start by presenting our model for conflict hypergraph for FK DCs and then use it to describe the solution for DCs. In short, our approach is to reverse-engineer $R_{1}$ from $V_{\text {Join }}$ so that joining it with $R_{2}$ recovers $V_{J o i n}$, and $R_{1}$ satisfies all DCs in $S_{D C}$.

\subsection{Conflict Hypergraphs and List Coloring}

We slightly augment the notion of conflict hypergraphs to illustrate possible Foreign Key DC violations caused by subsets of $R_{1}$ tuples.

Definition 5.1 (Conflict Hypergraph for Foreign Key DCs). A conflict hypergraph for $R_{1}$ and $S_{D C}$ is defined as $G=(V, E)$ where $V$ is the set of tuples in $R_{1}$ and $e=\left\{t_{1}, \ldots, t_{k}\right\} \in E$ if there is a Foreign Key DC of the form $\neg\left(\varphi\left(t_{1}, \ldots, t_{k}\right) \wedge t_{1} . F K=\ldots=t_{k} . F K\right)$ such that $\varphi\left(t_{1}, \ldots, t_{k}\right)$ evaluates to True.

It suffices to consider only $\varphi\left(t_{1}, \ldots, t_{k}\right)$ in the DCs when adding edges because $F K$ is initially missing. Abusing notation, we denote a set of tuples $\mathcal{T}$ violating $\varphi_{i}\left(t_{1}, \ldots, t_{k}\right)$ in a given $\mathrm{DC} \sigma_{i}$ by $\mathcal{T} \not_{\varphi_{i}} \sigma_{i}$ (we will use this notation in Algorithm 4).

Next, we give the connection between conflict hypergraph coloring and $F K$ assignment in $R_{1}$, so a proper coloring satisfies DCs.

Proposition 5.2. Given an instance of C-Extension, a coloring of the conflict hypergraph gives an assignment to all cells of the missing FK column in $R_{1}$ such that all DCs are satisfied.

We now turn to the problem of inferring $F K$ values in $R_{1}$ from the completed $V_{J o i n}$. Each tuple in $R_{1}$ can have multiple options for foreign key values that lead to $V_{\text {Join }}$ obtained in phase I. This establishes a list of possible colors, also referred to as candidate colors, for each vertex in the conflict graph. This problem is called List Coloring [2, 26]. It is a generalization of $k$-coloring, and is thus NP-hard. Hence, we use a heuristic approach, described by Algorithm 3, to color the vertices in a non-increasing order by degree, coloring as many vertices as possible. In Section 5.2, we describe how to color the vertices that remain uncolored by Algorithm 3 .

Algorithm 3 takes as input the conflict hypergraph $G_{c}$, a mapping $c$ from vertices to colors (initially empty) and a list of candidate colors $L$. It can be called on a graph with a partial color assignment (used in Algorithm 4 in Section 5.2). Initially, $s$ is an empty list 
that is used to store skipped vertices, and $l$ is the list of uncolored vertices sorted in non-increasing order by degree in $G_{c}$ (lines 2-3). In lines 4-12, we find a list of permissible colors for each $v \in l$, i.e., those vertices in $G_{c}$ that have not been given a color in the input color map $c$. If vertex $v$ belongs to an edge $e$ where all vertices other than $v$ in $e$ have the same color $c$, then $c$ is a forbidden color for $v$. Next, the algorithm assigns the "smallest" available color to $v$ in line 10. Otherwise, $v$ gets added to $s$ and remains uncolored (line 12). Finally, color map $c$ and list of skipped vertices $s$ are returned.

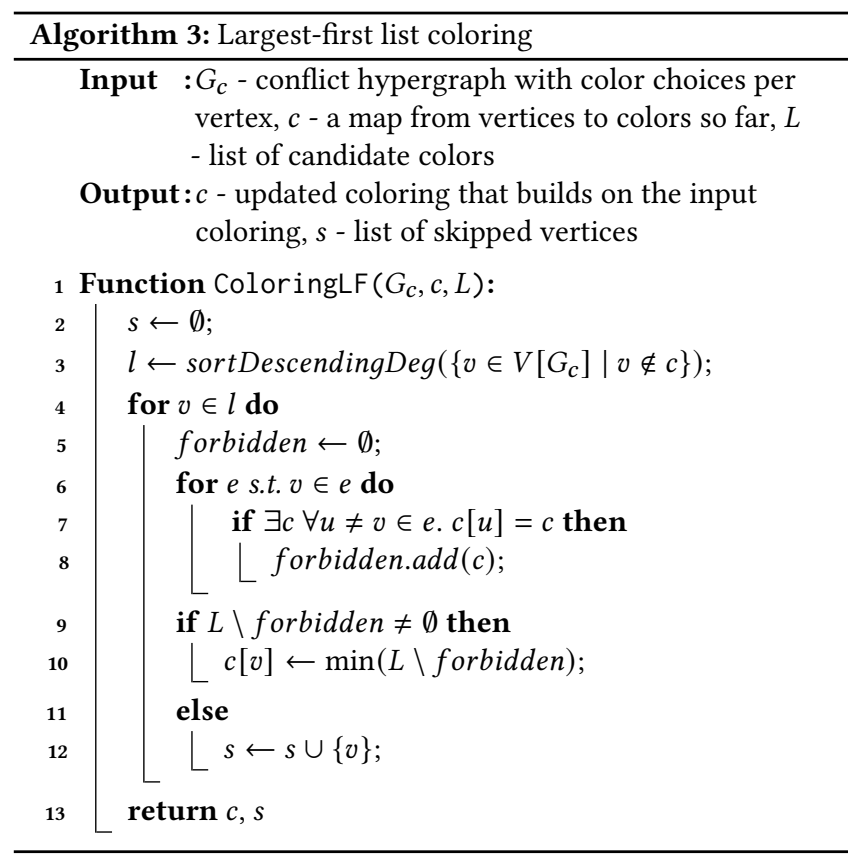

EXAMPLE 5.3. Reconsider the view $V_{\text {Join }}$ and DCs shown in Figure 5 and 2a, respectively. Figure 7 (including the dashed edges) gives the resulting conflict graph $G_{c}$. For example, there is an edge between vertices 1 and 2 because $t_{1} \cdot$ Rel $=t 2 . R e l=O w n e r$, so assigning them the same $F K$ value would violate $D C_{O, O}$. Here, $l=[2,1,3,4,8,9,5,6,7]$. Thus, Algorithm 3 returns: $c[1]=2, c[2]=1, c[3]=3, c[4]=$ $4, c[5]=3, c[6]=2, c[7]=2, c[8]=5$ and $c[9]=6$.

Complexity: The complexity of Algorithm 3 is $O(|V| \cdot \log |V|+$ $|V| \cdot|E|$ ) since, the algorithm sorts all vertices by degree (line 3) and then traverses all edges adjacent to each vertex.

\subsection{Algorithm for DCs}

We describe Algorithm 4 as the last step in solving C-Extension by completing $R_{1}$.FK. In Section 4 , we showed how to complete $V_{J o i n}$ by assigning values in the columns that came from $R_{2}$. For a tuple $t \in V_{\text {Join }}$ with values $t . B_{i}=b_{i}, 1 \leq i \leq q$, the candidate $F K$ values for the corresponding tuple in $R_{1}$ are given by $\pi_{K_{2}} \sigma_{B_{1}=b_{1}, \ldots, B_{q}=b_{q}} R_{2}$. We begin with an optimization that we employ in the algorithm. Optimization: Working with a single conflict hypergraph when $R_{1}$ contains a large number of tuples would not scale since the hypergraph can form one clique in the worst-case. However, observe that we can partition the filled-in $V_{\text {Join }}$ and $R_{2}$ by $B_{1}, \ldots, B_{q}$ values into sets, and only consider conflict hypergraphs within each set because the candidate $F K$ values are disjoint across sets.

EXAMPLE 5.4. Reconsider relations $R_{1}$ and $R_{2}$, and view $V_{\text {Join }}$ shown in Figures 1 and 5, respectively. In $V_{\text {Join }}$, the tuples have Area $=$ Chicago or Area $=$ NYC. Note that the set of candidate keys for tuples with Area $=$ Chicago comprises of values in $\pi_{h_{i d}} \sigma_{\text {Area }}=$ Chicago $R_{2}$ that is disjoint from those in $\pi_{h_{i d}} \sigma_{\text {Area }}=N Y C R_{2}$. This eliminates edges that would have been added to the conflict graph if we were to consider all vertices at once (shown as dashed edges in Figure 7). After partitioning $V_{\text {Join }}$ by $B_{1}, \ldots, B_{q}$ values and using the DCs in Figure $2 a$, we get two conflict graphs: (1) with vertices for tuples $t_{1}, \ldots, t_{7}$, and (2) with vertices for tuples $t_{8}$ and $t_{9}$. There is an edge between a pair of vertices when the corresponding tuples would violate a DC if assigned the same $h_{i d}$ value, and these are shown as solid edges in Figure 7.

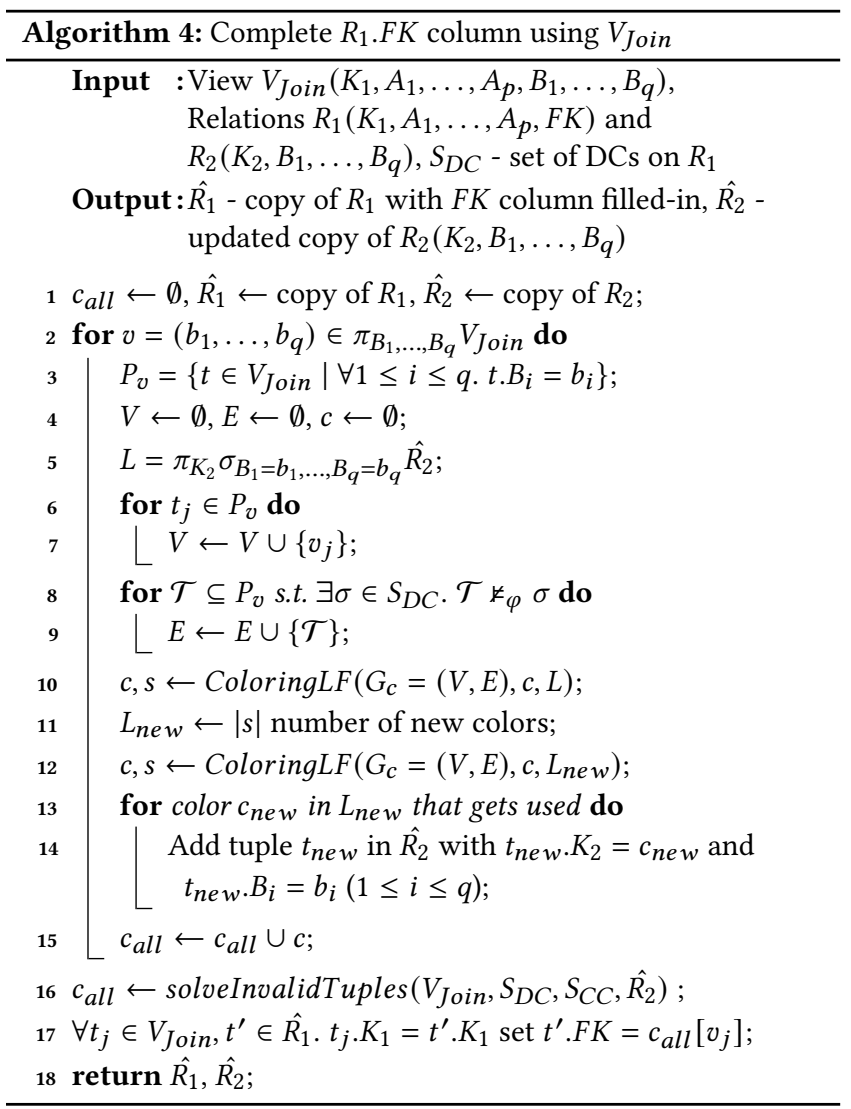

Algorithm 4 gets as input the view $V_{\text {Join }}$ outputted by the algorithm described in Section 4.3, relations $R_{1}$ (with missing $F K$ values) and $R_{2}$, and set $S_{D C}$. It outputs $\hat{R_{1}}$, i.e., $R_{1}$ with values in the $F K$ column and $\hat{R_{2}}$, i.e., $R_{2}$ with possible additional tuples (as described next). The algorithm can be divided into three parts: (1) coloring the tuples that were assigned $B_{1}, \ldots, B_{q}$ values in $V_{J o i n}$, (2) coloring the invalid tuples, i.e., tuples that were not assigned $B_{1}, \ldots, B_{q}$ values in $V_{\text {Join }}$, and (3) coloring any skipped tuples (defined in Section 5.1). Algorithm 4 maintains a map from tuples to their list of colors in $c$ and tracks the overall coloring in $c_{\text {all }}$. Eventually, $c_{\text {all }}$ has a color for every vertex that is used to complete $F K$ in $\hat{R_{1}}$ (lines 17,18 ). 
In lines 2-15, the algorithm iterates over each set of tuples with the same $B_{1}, \ldots, B_{q}$ value. Given a vector $v=\left(b_{1}, \ldots, b_{q}\right)$ of $q$ constants, it iterates over tuples in sets given by $P_{v}=\left\{t \in V_{\text {Join }} \mid \forall 1 \leq\right.$ $\left.i \leq q . t . B_{i}=b_{i}\right\}$. For each $P_{v}$, it generates the conflict hypergraph $G_{c}$ as follows: a node $v_{j}$ per tuple $t_{j}$, a list $L$ of candidate colors given by the keys from tuples in $\hat{R_{2}}$ with values $v_{j} . B_{1}, \ldots, v_{j} . B_{q}$, and an edge per set of tuples in $P_{v}$ that violates $\varphi$ in some DC. Next, $G_{c}, c$ and $L$ are inputted to Algorithm 3, which outputs a partial coloring $c$ and a list of skipped vertices $s$. Vertices in $s$ are colored using at most $|s|$ new colors (lines 11-14), resulting in insertion of tuples in $\hat{R_{2}}$ because colors correspond to primary keys in $\hat{R}_{2}$.

Procedure solveInvalidTuples (line 16) handles the invalid tuples (defined in Section 4.2). Since these do not have $B_{1}, \ldots, B_{q}$ values in $V_{\text {Join }}$, the corresponding $\hat{R_{1}}$ tuples are missing $F K$ values because we have not yet considered them in any conflict hypergraphs. We construct a hypergraph for tuples in $V_{\text {Join }}$ with edges incident to only the vertices for invalid tuples. However, the set $s$ outputted by Algorithm 3 may contain invalid tuples that had to be skipped for a lack of available colors. Our strategy for coloring these is to assign to each a combination of $B_{1}, \ldots, B_{q}$ values that minimizes the error stemming from the CCs (defined in Section 6), and generate a tuple in $\hat{R_{2}}$ with a fresh key and the chosen $B_{1}, \ldots, B_{q}$ values. Finally, $\hat{R_{1}} . F K$ values are assigned based on the coloring $c_{\text {all }}$ (line 17).

Proposition 5.5. Given $V_{\text {Join }}, R_{1}, R_{2}, S_{D C}$, Algorithm 4 outputs relations $\hat{R_{1}}, \hat{R_{2}}$ such that $\hat{R_{2}}$ is a copy of $R_{2}$, possibly with more tuples, and $\hat{R}_{1}$ is a copy of $R_{1}$ with all the values in the FK column completed such that $\forall \sigma \in S_{D C}, \hat{R_{1}} \vDash \sigma$, and $\hat{R_{1}} \bowtie_{F K=K_{2}} \hat{R_{2}}=V_{\text {Join }}$.

Complexity: The complexity of Algorithm 4 is $O\left(n \cdot\left|S_{D C}\right| \cdot\left(\begin{array}{l}n \\ T\end{array}\right)\right)$, where $\left|R_{1}\right|=n$, and $T$ is the number of tuples involved in the largest DC (assumed to be a constant), since the number of edges of each vertex can be at most $\left(\begin{array}{l}n \\ T\end{array}\right)$. The $n$ component stands for the possible need to iterate over all tuples in $V_{\text {Join }}$ in line 2, the $\left|S_{D C}\right|$ component stands for the possible need to iterate over all DCs when checking the condition in line 8 , and the $\left(\begin{array}{l}n \\ T\end{array}\right)$ component is added due to the need to iterate over all subsets of $P_{v}$ that may satisfy a DC in lines 8-9. Since Algorithm 3 (lines 10,12) has a complexity of $O\left(n \cdot\left(\begin{array}{c}n \\ T\end{array}\right)\right.$ ), and the loop (line 13) has complexity of $O(n)$, they are not presented in the overall complexity of the algorithm. Note that $\biguplus_{j=1}^{m} P_{v}^{j}=V_{\text {Join }}$, where $m$ is the number of iterations and $P_{v}^{j}$ is the set $P_{v}^{j}$ generated in iteration $j$.

Extending the solution to snowflake schemas: Our solution can be generalized to snowflake schemas in a manner similar to [5]. The idea is to start from the fact table (the central table) as $R_{1}$ and a table connected to it as $R_{2}$, i.e. going from the inside out in a Breadth-First Search manner. In every step, we include the previously completed tables in $R_{1}$, allowing CCs that span over the join view of multiple tables. This ensures that tuples are (possibly) added to the relation in the role of $R_{2}$ only once, since in the next step it would be considered as $R_{1}$ and thus maintain the foreign key dependency from the previous steps.

ExAmple 5.6. Consider a central Students table with two foreign key dependencies of a Majors table and a Courses table, and another foreign key connection to a Departments table through Majors:

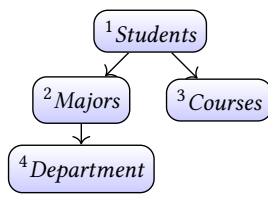

The steps of the algorithm are as follows:

\begin{tabular}{|c|c|c|}
\hline Step & $R_{1}$ & $R_{2}$ \\
\hline \hline 1 & Students & Majors \\
\hline 2 & Students $\bowtie$ Majors & Courses \\
\hline 3 & (Students $\bowtie$ Majors) $\bowtie$ Courses & Departments \\
\hline
\end{tabular}

At each step we can therefore consider CCs over all tables we have considered so far. For example, in step 2, we can consider CCs over ((Students $\bowtie$ Majors $) \bowtie$ Courses) and not just over Students $\bowtie$ Courses. Note that in the first step, we might have added artificial tuples to the Majors table. These are added without an FK value that connects them to the Departments table so we account for them in the last step of connecting the Majors and Departments tables, making sure that the DCs that apply to the Majors table are satisfied.

\section{EXPERIMENTS}

We analyze the performance of our (hybrid) approach, and compare it with a baseline algorithm (based on [5]) in these terms:

(1) Accuracy and runtime comparison between the baseline and our approach as data grows for fixed $S_{D C}$ and two settings of $S_{C C}$ (based on Section 4.2): (i) $S_{C C}$ with no intersecting CCs, and (ii) $S_{C C}$ with intersecting CCs.

(2) Accuracy and runtime comparison between the baseline and our approach for fixed data and combinations of good and bad $S_{D C}$ and $S_{C C}$. Good $S_{D C}$ creates zero cliques in conflict graphs and good $S_{C C}$ contains zero intersecting CCs.

(3) Runtime performance of our approach when data and $S_{D C}$ are kept fixed but the size of good $S_{C C}$ and bad $S_{C C}$ varies.

(4) Runtime performance of our approach for fixed data, and good $S_{D C}$ and $S_{C C}$ as the number of columns in $R_{2}$ grows.

We implemented our solution and baseline in Python 3.6.9 and Pandas DataFrame interface [39] on Tensor TXR231-1000R D126 Intel(R) Xeon(R) CPU E5-2640 v4 2.40GHz CPU with 512 GB (40 cores) of RAM. We use the standard PuLP [38] and NumPy Libraries for the ILP, and NetworkX [6] to construct and color conflict graphs. A summary of our findings:

(1) Our approach satisfies all CCs in the absence of intersecting CCs with no error. Additionally, our approach satisfies all DCs (as guaranteed by our theoretical analysis), whereas the baseline does not (Figures 8-10). Overall, our approach has the shortest runtime (Figure 11a) and achieves better accuracy for CCs and DCs together. Additionally, augmenting the input set of CCs with marginals over the non-key attributes in $R_{1}$ improves accuracy for CCs. We also find that the time spent by the baseline on the ILP solver alone is comparable to the total time taken by our approach for larger data scales.

(2) At a fixed data scale and for good and bad settings of DCs and CCs, where good DCs do not create cliques in conflict graphs and good CCs do not intersect, our approach has the shortest runtime. Its best performance is for good DCs and good CCs. In comparison, using bad DCs is slower because conflict graphs become denser, and using bad CCs is even slower because of the ILP solver. 
(3) Keeping the data and $S_{D C}$ fixed, we find that increasing the size of and/or intersections in $S_{C C}$ slows down $V_{\text {Join }}$ completion, increasing the runtime of our approach (Figure 13)

(4) We find that the time spent on coloring grows faster than that for recursing on Hasse diagrams as the number of columns in $R_{2}$ grows when good $S_{D C}$ and $S_{C C}$ are used.

\subsection{Setup}

We now describe the experimental setup (summarized in Table 3) and define the error measures that are used to evaluate accuracy. We vary the database size (Table 1), DCs and CCs (see [21]) to examine the scalability and accuracy of our solution. In Section 6.2, the errors and runtimes are averaged over 3 independent runs. Data. We perform experiments on a dataset that is derived from the 2010 U.S. Decennial Census [45] comprising of two relations Persons $\left(p_{i d}\right.$, Rel, Age, Multi-ling, $\left.h_{i d}\right)$ and Housing $\left(h_{i d}\right.$, Tenure, Area), with Persons $\left(R_{1}\right)$ missing all values in its foreign key column $h_{i d}$. The different data scales are given in Table 1 . By construction, $V_{J o i n}$ and Persons contain the same number of tuples. We also consider up to 10 (non-key) columns in Housing, where we go from (Tenure, Area) to (Tenure, County, Area, St), add (Div, Reg) and then add binary attributes (Water, Bath) followed by (Fridge, Stove). Note that values in Div and Reg are determined by the $S t$ value.

Table 1: Data scales given by the number of tuples

\begin{tabular}{|c|c|c|c|}
\hline Scale & Persons table & Housing table & $V_{\text {Join }}$ \\
\hline $1 \times$ & 25,099 & 9,820 & 25,099 \\
\hline $2 \times$ & 50,039 & 19,640 & 50,039 \\
\hline $5 \times$ & 124,746 & 49,100 & 124,746 \\
\hline $10 \times$ & 249,259 & 98,200 & 249,259 \\
\hline $40 \times$ & $1,015,686$ & 392,800 & $1,015,686$ \\
\hline $80 \times$ & $2,043,975$ & 785,600 & $2,043,975$ \\
\hline $120 \times$ & $3,064,328$ & $1,178,400$ & $3,064,328$ \\
\hline $160 \times$ & $4,097,471$ & $1,571,200$ & $4,097,471$ \\
\hline
\end{tabular}

Denial Constraints. $S_{D C}^{\text {all }}$ is the set of DCs (see [21]) that not only gives the permissible age gap between a homeowner ( $R e l=$ Owner) and other members in the same home, but also limits the number of homeowners, spouses and unmarried partners per home. $S_{D C}^{\text {good }}$ contains first $8 \mathrm{DCs}$, none of which create cliques in conflict graphs. Cardinality Constraints. We use two sets of CCs, $S_{C C}^{g o o d}$ and $S_{C C}^{b a d}$, with 1001 CCs each (see [21]). We assume that each input CC specifies a condition on an attribute from both $R_{1}$ and $R_{2} . S_{C C}^{b a d}$ contains CCs with intersecting Age intervals, but $S_{C C}^{\text {good }}$ does not. Error Measures. We measure relative CC error $\operatorname{err}_{i}$ as $\frac{\left|\hat{c_{i}}-c_{i}\right|}{\max \left(10, c_{i}\right)}$, where $\hat{c_{i}}$ and $c_{i}$ are $C C_{i}$ 's (in $S_{C C}$ ) counts in the solution and input. We use a threshold of 10 in the denominator because some CCs have a target count of 0 for small data scales. We report the median relative CC errors in Figures 8-10, where $y=1$ represents $100 \%$ error. We measure $D C$ error as the fraction of tuples in $\hat{R}_{1}$ that violate a $D C \in S_{D C}$. E.g., if $h_{i d}$ value in the first two tuples in Persons relation in Figure 3 was 2, then the DC error would be 2/9. Baseline. Arasu et al. [5] focuses on the generation of synthetic databases with snowflake schema, where all joins are foreign key joins. This work considers CCs alone (no DCs) and imputes FK using $V_{\text {Join }}$. Motivated by this work, we establish the two baseline versions given below (Section 7 surveys more related works).

(1) Baseline: First, we use Algorithm 1 (without the for loop on line 9) to fill-in tuples in $V_{\text {Join }}$. Any $V_{\text {Join }}$ tuple without an assignment is completed by randomly assigning values in $B_{1}, \ldots, B_{q}$. In phase II, we randomly assign a value from the candidate $F K$ values given by $V_{\text {Join }}$ for each tuple in $R_{1}$.

(2) Baseline with marginals: We also study the impact of augmenting $S_{C C}$ with all Age-Rel-Multi-ling (all-way) marginals from Persons, where domains of numerical attributes are broken using intervalization [5] on $S_{C C}$. Note that the marginals have equal target counts in Persons and $V_{\text {Join }}$ by construction. They ensure that each variable participates in the ILP, and is thus assigned a value in the solution. We find that this fills in all $V_{\text {Join }}$ tuples. We refer to this algorithm as baseline with marginals that uses Algorithm 1 for phase I, followed by random assignment in $F K$ using $V_{\text {Join }}$ for phase II. Hence, it falls in-between the baseline and our approach (Section 4.3).

Table 2: Datasets used in experiments, with details about the data scales, DCs and CCs given in Table 1 and in [21]

\begin{tabular}{|c|c|c|c|}
\hline Dataset no. & Data Scale & DCs & CCs \\
\hline $1-5$ & $1 \times$ to $40 \times$ & $S_{D C}^{\text {all }}$ & $S_{C C}^{\text {good }}$ \\
\hline $6-10$ & $1 \times$ to $40 \times$ & $S_{D C}^{\text {all }}$ & $S_{C C}^{\text {bad }}$ \\
\hline 11 & $10 \times$ & $S_{D C}^{\text {good }}$ & $S_{C C}^{\text {good }}$ \\
12 & $10 \times$ & $S_{D C}^{\text {good }}$ & $S_{C C}^{\text {bad }}$ \\
\hline $13-17$ & $10 \times$ & $S_{D C}^{\text {all }}$ & $(500,600,700,800,900) S_{C C}^{\text {good }}$ \\
\hline $18-22$ & $10 \times$ & $S_{D C}^{\text {all }}$ & $(500,600,700,800,900) S_{C C}^{\text {bad }}$ \\
\hline $23-26$ & $40 \times$ to $160 \times$ & $S_{D C}^{\text {good }}$ & $S_{C C}^{\text {good }}$ \\
\hline $27-30$ & $40 \times$ to $160 \times$ & $S_{D C}^{\text {good }}$ & $S_{C C}^{\text {bad }}$ \\
\hline $31-34$ & $10 \times(4,6,8,10$ non- & $S_{D C}^{\text {good }}$ & $S_{C C}^{\text {good }}$ \\
\hline
\end{tabular}

Table 3: Experimental settings for Figures 8-13. Table 2 contains details about the input datasets

\begin{tabular}{|c|c|c|c|}
\hline Experiment & Figure & Algorithm & Input datasets \\
\hline \hline \multirow{4}{*}{ Accuracy Exp. } & $8 \mathrm{a}$ & Baselines vs Hybrid & $1-5$ \\
\cline { 2 - 4 } & $8 \mathrm{~b}$ & Baselines vs Hybrid & $6-10$ \\
\cline { 2 - 4 } & 9 & Baselines vs Hybrid & 10 \\
\cline { 2 - 4 } & 10 & Baselines vs Hybrid & $11,12,4,9$ \\
\hline \hline \multirow{3}{*}{ Scalability Exp. } & $11 \mathrm{a}$ & Baselines vs Hybrid & 9,10 \\
\cline { 2 - 4 } & $11 \mathrm{~b}$ & Hybrid & $11,23-26,12,27-30$ \\
\cline { 2 - 4 } & 12 & Hybrid & $11,31-34$ \\
\cline { 2 - 4 } & 13 & Hybrid & 17,22 \\
\hline
\end{tabular}

\subsection{Experimental Findings}

We discuss results and address aspects raised at the start of Section 6. Our approach vs baselines - Accuracy. We consider the experimental setup from Table 3 for the accuracy experiments, and detail our results in Figures 8-10. Our approach always satisfies all DCs, and all CCs in $S_{C C}^{g o o d}$. For $S_{C C}^{b a d}$ (Table $8 \mathrm{~b}$ ), the median CC error is 0 but the smallest and largest average errors are 0.048 and 0.093 due to limitations in augmenting $S_{C C}$ (Section 4.3). In contrast, the baseline gives median CC and DC errors between 0.233-0.580 and $0.228-0.373$, whereas baseline with marginals satisfies all CCs but gives DC errors between 0.402-0.510. We take a closer look at the relative CC errors for data Scale $40 \times$ and $S_{C C}^{b a d}$ in Figure 9. Note that DCs are used only after $V_{J o i n}$ is partitioned by $B_{1}, \ldots, B_{q}$ values, so CCs affect the quality of the solution given by Algorithm 4 .

Next, we look at combinations of good and bad cases of DCs and CCs for data at Scale 10×. Again, our approach satisfies all DCs and gives a median CC error of 0 . Here, half the CCs were passed into Algorithm 2 that satisfies CCs exactly. The remaining CCs are augmented (Section 4.3) to improve accuracy for Algorithm 1. We 


\begin{tabular}{|c|c|c|c|c|c|c|}
\hline Scale & \multicolumn{3}{|c|}{ CC error } & \multicolumn{3}{c|}{ DC error } \\
& Baseline & $\begin{array}{c}\text { Baseline } \\
\text { (marginals) }\end{array}$ & Hybrid & Baseline & $\begin{array}{c}\text { Baseline } \\
\text { (marginals) }\end{array}$ & Hybrid \\
\hline $1 \times$ & 0.300 & 0 & 0 & 0.218 & 0.445 & 0 \\
\hline $2 \times$ & 0.367 & 0 & 0 & 0.245 & 0.465 & 0 \\
\hline $5 \times$ & 0.526 & 0 & 0 & 0.274 & 0.446 & 0 \\
\hline $10 \times$ & 0.604 & 0 & 0 & 0.303 & 0.489 & 0 \\
\hline $40 \times$ & 0.559 & 0 & 0 & 0.371 & 0.520 & 0 \\
\hline
\end{tabular}

(a) $S_{D C}^{\text {all }}, S_{C C}^{\text {good }}$

\begin{tabular}{|c|c|c|c|c|c|c|}
\hline Scale & Baseline & $\begin{array}{c}\text { CC error } \\
\text { Baseline } \\
\text { (marginals) }\end{array}$ & Hybrid & Baseline & $\begin{array}{c}\text { DC error } \\
\text { Baseline } \\
\text { (marginals) }\end{array}$ & Hybrid \\
\hline $1 \times$ & 0.233 & 0 & 0 & 0.228 & 0.435 & 0 \\
\hline $2 \times$ & 0.300 & 0 & 0 & 0.246 & 0.434 & 0 \\
\hline $5 \times$ & 0.467 & 0 & 0 & 0.279 & 0.402 & 0 \\
\hline $10 \times$ & 0.537 & 0 & 0 & 0.305 & 0.510 & 0 \\
\hline $40 \times$ & 0.580 & 0 & 0 & 0.373 & 0.489 & 0 \\
\hline
\end{tabular}

(b) $S_{D C}^{a l l}, S_{C C}^{b a d}$

Figure 8: Error rate comparison between the baseline, baseline with marginals and hybrid as data grows from Scale $1 \times-40 \times$ and: (a) $S_{D C}^{\text {all }}(12 \mathrm{DCs})$ and $S_{C C}^{\text {good }}(1001 \mathrm{CCs})$ are used, and (b) $S_{D C}^{\text {all }}(12 \mathrm{DCs})$ and $S_{C C}^{b a d}(1001 \mathrm{CCs})$ are used

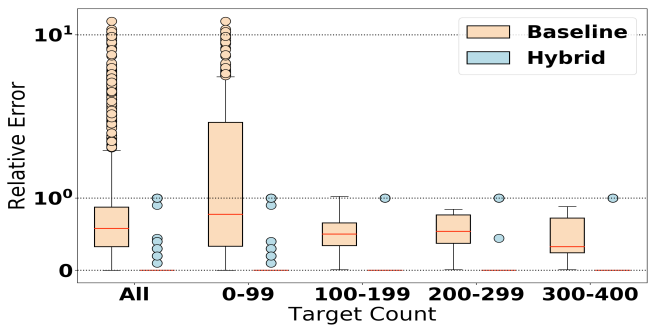

Figure 9: Relative CC error incurred by the baseline and hybrid for data Scale $40 \times, S_{D C}^{\text {all }}(12 \mathrm{DCs})$ and $S_{C C}^{b a d}(1001 \mathrm{CCs})$. We omit baseline with marginals as it satisfies all CCs

\begin{tabular}{|c|c|c|c|c|c|c|}
\hline $\begin{array}{c}\text { Data- } \\
\text { set }\end{array}$ & $\begin{array}{c}\text { Baseline } \\
\text { (no aug) }\end{array}$ & $\begin{array}{c}\text { CC error } \\
\text { Baseline } \\
\text { (with aug) }\end{array}$ & Hybrid & $\begin{array}{c}\text { Baseline } \\
\text { (no aug) }\end{array}$ & $\begin{array}{c}\text { Baseline } \\
\text { (with aug) }\end{array}$ & Hybrid \\
\hline 11 & 0.618 & 0 & 0 & 0.081 & 0.009 & 0 \\
\hline 12 & 0.573 & 0 & 0 & 0.079 & 0.004 & 0 \\
\hline 4 & 0.604 & 0 & 0 & 0.303 & 0.489 & 0 \\
\hline 9 & 0.537 & 0 & 0 & 0.305 & 0.510 & 0 \\
\hline
\end{tabular}

Figure 10: CC and DC error comparison between baseline, baseline with marginals and hybrid for combinations of good and bad cases of DCs and CCs at data Scale $10 \times$

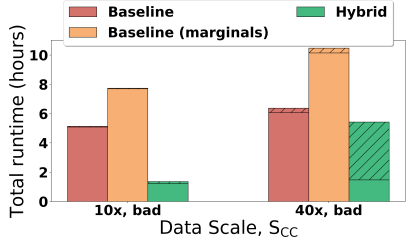

(a) $S_{D C}^{\text {all }}(12 \mathrm{DCs}), S_{C C}^{\text {bad }}$

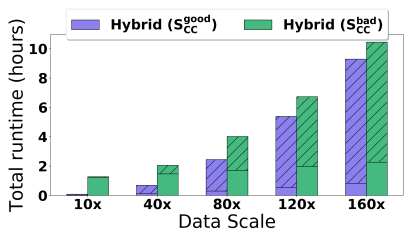

(b) $S_{D C}^{\text {good }}$ (8 DCs), $S_{C C}^{\text {good }}$ or $S_{C C}^{b a d}$ Figure 11: Shaded area depicts phase II in: (a) Runtime comparison between baseline and hybrid for $S_{D C}^{\text {all }}, S_{C C}^{b a d}(1001 \mathrm{CCs})$ and data Scale $10 \times$ or $40 \times$, (b) Runtime of hybrid for $S_{D C}^{\text {good }}$ and data Scales $10 \times-160 \times$ with $S_{C C}^{\text {good }}$ or $S_{C C}^{b a d}(1001$ CCs)

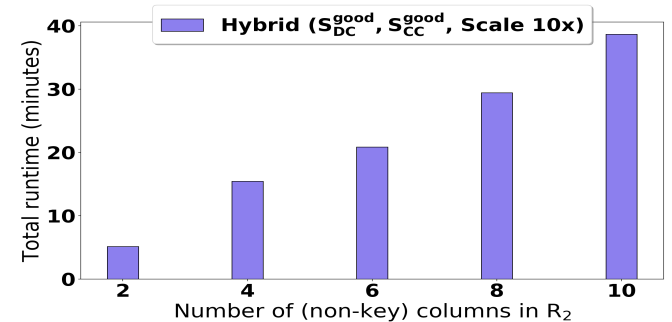

Figure 12: Runtime of hybrid for $S_{D C}^{\text {good }}$ (8 DCs), $S_{C C}^{\text {good }}(1001$ CCs) and data Scale $10 \times$ as number of columns in $R_{2}$ grows find that most CCs have a relative error of 0 . For $S_{C C}^{b a d}$, the average $\mathrm{CC}$ error given by our approach is 0.0735 . In contrast, baseline gives CC errors between $0.537-0.618$ and DC errors between 0.079-0.305, whereas baseline with marginals satisfies all CCs but gives DC errors between 0.004-0.510 due to random assignment in $R_{1}$.FK.

Our approach vs baselines - Runtime. We consider the experimental setup as given in Table 3 for the scalability experiments. The total runtimes at data Scales $10 \times$ and $40 \times$ are given in Figure 11a. Observe that the time spent on phase II by the baseline is minimal because it randomly assigns $F K$ values, whereas our approach colors conflict graphs to satisfy all DC exactly. In our approach, Algorithm 1 and 4 are the bottlenecks taking $25.23 \%$ and $72.74 \%$ of the total runtime for $S_{D C}^{a l l}$ and $S_{C C}^{b a d}$ at data Scale $40 \times$.

At data Scale $40 \times$, the runtimes for completing $V_{\text {Join }}$ for $S_{C C}^{\text {good }}$ and $S_{C C}^{b a d}$ are as follows: (1) baseline takes 5.88 hours and 6.07 hours, (2) baseline with marginals takes 9.75 hours and 10.15 hours, and (3) our approach takes 7.79 minutes and 1.48 hours. The corresponding total runtimes are: (1) 6.19 hours and 6.38 hours, (2) 10.04 hours and 10.49 hours, and (3) 1.06 hours and 5.43 hours hours. Our approach has the shortest runtime in completing $V_{\text {Join }}$ because we take advantage of the relationships between the CCs to separate out intersecting CCs from $S_{C C}$ which reduces the time to solve the ILP. In contrast, the baseline creates one large ILP with all CCs (with or without all-way marginals). In addition, our approach does not need the ILP solver for $S_{C C}^{g o o d}$, further improving the runtime.

Note that the baselines do not take into account the DCs and randomly assign $F K$ values based on filled-in $V_{\text {Join }}$, so solving the ILP dominates the time to populate $h_{i d}$ in Persons $\left(R_{1}\right)$. Our approach has the shortest runtime for $\left(S_{D C}^{\text {good }}, S_{C C}^{\text {good }}\right)$ (at 5.17 minutes), followed by $\left(S_{D C}^{\text {all }}, S_{C C}^{\text {good }}\right),\left(S_{D C}^{\text {good }}, S_{C C}^{\text {bad }}\right)$ and $\left(S_{D C}^{\text {all }}, S_{C C}^{b a d}\right)$ (at 1.36 hours). Intuitively, for fixed data, completing $V_{J o i n}$ is faster for $S_{C C}^{\text {good }}$ and conflict graphs are more likely to have fewer edges for $S_{D C}^{\text {good }}$. For $\left(S_{D C}^{\text {good }}, S_{C C}^{\text {good }}\right)$ and $\left(S_{D C}^{\text {all }}, S_{C C}^{\text {bad }}\right):(1)$ baseline took 4.84-5.14 hours, and (2) baseline with marginals took close to 8 hours. Baseline ran faster because it runs the ILP solver without the marginals.

Larger data scales - Runtime. We examine our solution's runtime for larger data scales when $S_{D C}^{\text {good }}$ is used with $S_{C C}^{\text {good }}$ vs $S_{C C}^{b a d}$ (see Figure 11b). We find that our solution scales well, taking a total of 9.3 hours for $S_{C C}^{g o o d}$ and 10.46 hours for $S_{C C}^{b a d}$ at data Scale $160 \times$. Increasing the number of $R_{2}$ columns - Runtime. We study the effect of increasing the number of $R_{2}$ columns on the runtime of our approach for $S_{D C}^{\text {good }}, S_{C C}^{\text {good }}$ and data Scale $10 \times$ (see Figure 12). We describe in Section 6.1 how the number of columns in $R_{2}$ grows from 
2 to 10. Our approach takes a total of 5.17 minutes for 2 columns and 38.66 minutes for 10 columns because we only consider the columns that are used in $S_{C C}$ for combo $o_{\text {unused }}$ (Algorithm 2).

Increasing the number of CCs - Runtime and accuracy. Here we study the effect of the size of $S_{C C}$ on the runtime and error in our approach (the last row in Table 3). The breakdown of runtimes for 900 CCs chosen from $S_{C C}^{g o o d}$ and $S_{C C}^{b a d}$ is given in Figure 13.

\begin{tabular}{|c|c|c|c|c|}
\cline { 2 - 5 } \multicolumn{1}{c|}{} & \multicolumn{2}{c|}{900 CCs from $S_{C C}^{\text {good }}$} & \multicolumn{2}{c|}{900 CCs from $S_{C C}^{\text {bad }}$} \\
\cline { 2 - 5 } \multicolumn{1}{c|}{} & Time & $\%$ & Time & $\%$ \\
\hline $\begin{array}{c}\text { Pairwise } \\
\text { Comparison }\end{array}$ & $4.48 \mathrm{~s}$ & 1.12 & $4.24 \mathrm{~s}$ & 0.10 \\
\hline Recursion & $1.70 \mathrm{~m}$ & 25.64 & $1.29 \mathrm{~m}$ & 1.76 \\
\hline ILP solver & - & - & $1.06 \mathrm{~h}$ & 86.21 \\
\hline Coloring & $4.87 \mathrm{~m}$ & 73.24 & $8.77 \mathrm{~m}$ & 11.93 \\
\hline
\end{tabular}

Figure 13: Runtime breakdown of the hybrid approach for data Scale $10 \times$ with $S_{D C}^{\text {all }}(12 \mathrm{DCs})$ and $900 \mathrm{CCs}$ from $S_{C C}^{\text {good }}$ or $S_{C C}^{b a d}$ (overall we have 1001 CCs for both)

As more CCs are used, the time spent on labeling pairs of CCs as disjoint, contained or intersecting increases. Since $S_{C C}^{\text {good }}$ contains no intersecting CCs, the ILP solver is not used and the runtime is faster. Algorithm 2 takes 1.42 minutes for $500 \mathrm{CCs}$ and 1.78 minutes for $900 \mathrm{CCs}$. More CCs not only cause more updates to $V_{\text {Join }}$, but may also add CCs where Area is used without Tenure, creating tuples with a partial assignment in $V_{\text {Join }}$ that are completed in line 17 of the algorithm. For $900 \mathrm{CCs}$, the total runtime is 6.65 minutes, of which 4.87 minutes are spent in filling-in $R_{1}$ (Algorithm 4).

When more CCs are chosen from $S_{C C}^{b a d}$, we see an increase in the number of CCs passed to Algorithm 1 that makes the ILP solver slower. Algorithm 2 takes 1.21 minutes for $500 \mathrm{CCs}$ and 1.36 minutes for $900 \mathrm{CCs}$, whereas Algorithm 1 takes 25.99 minutes for $500 \mathrm{CCs}$ and 1.06 hours for $900 \mathrm{CCs}$. For $900 \mathrm{CCs}$, the total runtime is 1.23 hours, of which 8.77 minutes are spent in completing $R_{1}$.

Our approach satisfies all DCs, and CCs in $S_{C C}^{\text {good }}$. The median and average $\mathrm{CC}$ error rates are 0 and 0.034-0.092, resp. We give an optimization for (bottleneck) coloring step in the full version [21].

\section{RELATED WORK}

Data generation has been the focus of multiple works, e.g., [4, 5 , $9,11,12,19,22,25,32,35,42,44,46,51]$. The main novelty of this paper is the generation of foreign keys for existing database relations while reducing the error of a set of CCs and ensuring the satisfaction of a set of DCs that relate to the foreign key attribute.

A prominent line of work uses CCs to define the desired parameters of the generated data [5, 9, 44]. QAGen [9] was among the first system that focused on data generation in a query-aware fashion. The target application was to test the performance of a database management system (DBMS) when given a database schema, one parametric Conjunctive Query and a collection of constraints on each operator. MyBenchmark [32] extends [9] by generating a set of database instances that approximately satisfies the cardinality expectations from a set of query results. HYDRA [44] uses a declarative approach that allows for the generation of a database summary that can be used for dynamically generating data for query execution. Arasu et. al. [5] proposed a framework that supports multiple CCs and generates data using a graphical model that converts the $\mathrm{CCs}$ to equations, using the concept of intervalization for efficient computations. Indeed, we have drawn on this work for Algorithm 1. These approaches allow for complex CCs, whereas our approach allows for DCs as well. A recent work [48] has proposed a solution for generating multiple data samples using a seed sample of the data (generated by previous work [49]), statistical constraints and data validity constraints specified in OCL [1]. UpSizeR [51] has focused on scaling the database while maintaining foreign key constraints. Data generation from the database schema and statistical information has also been studied [42, 46].

The field of data privacy [17, 28, 30, 34, 36, 50, 54] typically gives mechanisms that generate query answers that do not expose features of the underlying private data, rather than generate the data itself. Some works $[23,56]$ focus on providing consistent query answers, but none, to our knowledge, consider queries over linked data that guarantee the satisfaction of a set of ICs. Yahalom et. al. [55] developed a framework for converting production data into test data by modeling it as a constrained satisfaction problem (CSP) using specific constraints that can be expressed as part of the CSP.

Finally, DCs (without CCs) have been mainly explored in relation with data cleaning $[3,8,14,16,18,20,27,43]$. Previous work on the subject has focused on two main approaches: (1) repairing attribute values in cells $[8,16,43]$ and (2) tuple deletion [14, 20,33]. In this context, there has been previous work on automatically discovering DCs from the complete data [15, 31, 40]. We consider DCs based on the FK column, which is missing. In many scenarios, as is the premise in many data cleaning works (e.g., [14, 16, 20, 43]), such DCs can be naturally inferred from the schema or from domain knowledge. As in data cleaning, the constraints can be formulated by the users as logical statements [43] or as SQL queries [20].

\section{CONCLUSIONS AND LIMITATIONS}

We have defined the problem of generating links between database relations using linear CCs and foreign key DCs, and proved that it is intractable. Therefore, we have shown a novel two-phase heuristic solution. Our solution first considers the CCs, with a hybrid approach that combines an ILP-based solution and a solution based on specific relationships between the CCs. Second, our approach utilizes a version of conflict graph coloring in order to find a completion of the tuples that satisfies all DCs. Our experimental results show that our solution is both accurate and scalable.

There are many intriguing directions for future work. First, our solution focuses on linear CCs and a subset of DCs. Finding a solution when the constraints include non-linear CCs (e.g., CCs on the number of rows that share the same foreign key) and general DCs (e.g., DCs on tuples that do not share a foreign key) is an important extension of our approach. Second, in phase I, we assume foreign key dependence that induces a one-to-one mapping between the tuples of $R_{1}$ and the tuples of $V_{\text {Join }}$. Examining other join dependencies that do not have this property is an interesting direction of exploration. Third, in phase II, tuples may be artificially added to $R_{2}$ due to the coloring algorithm. Some scenarios may not allow such augmentation and thus require different solutions. Finally, the extension of our solution to non-relational databases, such as graph databases and wide-column store is another subject of future study. Acknowledgements. This work was supported by the National Science Foundation under grants 1408982 and 1703431; and by DARPA and SPAWAR under contract N66001-15-C-4067. 


\section{REFERENCES}

[1] 2017. Object Constraint Language 2.4 Specification. https://www.omg.org/spec/ OCL/About-OCL/.

[2] Dimitris Achlioptas and Michael Molloy. 1997. The Analysis of a List-Coloring Algorithm on a Random Graph (extended abstract. IEEE (1997). https://users. soe.ucsc.edu/ optas/papers/list-coloring.pdf

[3] Foto N. Afrati and Phokion G. Kolaitis. 2009. Repair Checking in Inconsistent Databases: Algorithms and Complexity. In ICDT. 31-41.

[4] Shaukat Ali, Muhammad Zohaib Z. Iqbal, Andrea Arcuri, and Lionel C. Briand 2013. Generating Test Data from OCL Constraints with Search Techniques. IEEE Trans. Software Eng. 39, 10 (2013), 1376-1402.

[5] Arvind Arasu, Raghav Kaushik, and Jian Li. 2011. Data Generation Using Declarative Constraints. In SIGMOD. 685-696.

[6] Daniel A. Schult Aric A. Hagberg and Pieter J. Swart. 2008. Exploring network structure, dynamics, and function using NetworkX. SciPy.

[7] Boaz Barak, Kamalika Chaudhuri, Cynthia Dwork, Satyen Kale, Frank McSherry, and Kunal Talwar. 2007. Privacy, accuracy, and consistency too: a holistic solution to contingency table release. In SIGACT-SIGMOD-SIGART. 273-282.

[8] Leopoldo E. Bertossi, Solmaz Kolahi, and Laks V. S. Lakshmanan. 2013. Data Cleaning and Query Answering with Matching Dependencies and Matching Functions. Theory Comput. Syst. 52, 3 (2013), 441-482.

[9] Carsten Binnig, Donald Kossmann, Eric Lo, and M. Tamer Özsu. 2007. QAGen: generating query-aware test databases. In SIGMOD. 341-352.

[10] Philip Bohannon, Wenfei Fan, Floris Geerts, Xibei Jia, and Anastasios Kementsietsidis. 2007. Conditional functional dependencies for data cleaning. In ICDE 746-755.

[11] Nicolas Bruno and Surajit Chaudhuri. 2005. Flexible Database Generators. In Proc. VLDB Endow. 1097-1107.

[12] Teodora Sandra Buda, Thomas Cerqueus, John Murphy, and Morten Kristiansen. 2013. VFDS: Very fast database sampling system. In IRI. 153-160.

[13] Surajit Chaudhuri and Umeshwar Dayal. 1997. An Overview of Data Warehousing and OLAP Technology. SIGMOD Rec. 26, 1 (March 1997), 65-74.

[14] Jan Chomicki and Jerzy Marcinkowski. 2005. Minimal-change integrity maintenance using tuple deletions. Inf. Comput. 197, 1-2 (2005), 90-121.

[15] Xu Chu, Ihab F. Ilyas, and Paolo Papotti. 2013. Discovering Denial Constraints. PVLDB 6, 13 (2013), 1498-1509.

[16] Xu Chu, Ihab F. Ilyas, and Paolo Papotti. 2013. Holistic data cleaning: Putting violations into context. In ICDE. 458-469.

[17] Cynthia Dwork. 2006. Differential Privacy. In ICALP, Vol. 4052. 1-12.

[18] Ronald Fagin, Benny Kimelfeld, and Phokion G. Kolaitis. 2015. Dichotomies in the Complexity of Preferred Repairs. In PODS. 3-15.

[19] Bálint Fazekas and Attila Kiss. 2018. Statistical Data Generation Using Sample Data. In New Trends in Databases and Information Systems, Vol. 909. 29-36.

[20] Amir Gilad, Daniel Deutch, and Sudeepa Roy. 2020. On Multiple Semantics for Declarative Database Repairs. In SIGMOD. 817-831.

[21] Amir Gilad, Shweta Patwa, and Ashwin Machanavajjhala. 2021. Synthesizing Linked Data Under Cardinality and Integrity Constraints. https://arxiv.org/abs/ 2103.14435. CoRR abs/2103.14435 (2021).

[22] Jim Gray, Prakash Sundaresan, Susanne Englert, Kenneth Baclawski, and Peter J Weinberger. 1994. Quickly Generating Billion-Record Synthetic Databases. In SIGMOD. 243-252.

[23] Michael Hay, Vibhor Rastogi, Gerome Miklau, and Dan Suciu. 2010. Boosting the Accuracy of Differentially Private Histograms Through Consistency. Proc. VLDB Endow. 3, 1 (2010), 1021-1032.

[24] Xi He, Ashwin Machanavajjhala, and Bolin Ding. 2014. Blowfish privacy: tuning privacy-utility trade-offs using policies. In SIGMOD. ACM, 1447-1458.

[25] Kenneth Houkjær, Kristian Torp, and Rico Wind. 2006. Simple and Realistic Data Generation. In Proc. VLDB Endow. 1243-1246.

[26] Tommy R Jensen and Bjarne Toft. 2011. Graph coloring problems. Vol. 39. John Wiley \& Sons.

[27] Solmaz Kolahi and Laks V. S. Lakshmanan. 2009. On approximating optimum repairs for functional dependency violations. In ICDT. 53-62.

[28] Ios Kotsogiannis, Yuchao Tao, Xi He, Maryam Fanaeepour, Ashwin Machanavajjhala, Michael Hay, and Gerome Miklau. 2019. PrivateSQL: A Differentially Private SQL Query Engine. Proc. VLDB Endow. 12, 11 (2019), 1371-1384.

[29] Chao Li, Michael Hay, Gerome Miklau, and Yue Wang. 2014. A Data- and Workload-Aware Query Answering Algorithm for Range Queries Under Differential Privacy. Proc. VLDB Endow. 7, 5 (2014), 341-352.
[30] Chao Li, Gerome Miklau, Michael Hay, Andrew McGregor, and Vibhor Rastogi. 2015. The matrix mechanism: optimizing linear counting queries under differential privacy. VLDB f. 24, 6 (2015), 757-781.

[31] Ester Livshits, Alireza Heidari, Ihab F. Ilyas, and Benny Kimelfeld. 2020. Approximate Denial Constraints. Proc. VLDB Endow. 13, 10 (2020), 1682-1695.

[32] Eric Lo, Nick Cheng, and Wing-Kai Hon. 2010. Generating Databases for Query Workloads. Proc. VLDB Endow. 3, 1 (2010), 848-859.

[33] Andrei Lopatenko and Leopoldo E. Bertossi. 2007. Complexity of Consistent Query Answering in Databases Under Cardinality-Based and Incremental Repair Semantics. In ICDT. 179-193.

[34] Ashwin Machanavajjhala, Daniel Kifer, Johannes Gehrke, and Muthuramakrishnan Venkitasubramaniam. 2007. $L$-diversity: Privacy beyond $k$-anonymity. $A C M$ Trans. Knowl. Discov. Data 1, 1 (2007), 3.

[35] Heikki Mannila and Kari-Jouko Räihä. 1989. Automatic Generation of Test Data for Relational Queries. f. Comput. Syst. Sci. 38, 2 (1989), 240-258.

[36] Ryan McKenna, Gerome Miklau, Michael Hay, and Ashwin Machanavajjhala. 2018. Optimizing error of high-dimensional statistical queries under differential privacy. Proc. VLDB Endow. 11, 10 (2018), 1206-1219.

[37] Ryan McKenna, Daniel Sheldon, and Gerome Miklau. 2019. Graphical-model based estimation and inference for differential privacy. In ICML, Vol. 97. 44354444.

[38] Stuart Mitchell, Stuart Mitchell Consulting, and Iain Dunning. 2011. PuLP: A Linear Programming Toolkit for Python. http://www.optimization-online.org/ DB_FILE/2011/09/3178.pdf.

[39] The pandas development team. 2020. pandas-dev/pandas: Pandas. https://doi. org/10.5281/zenodo.3509134

[40] Eduardo H. M. Pena, Eduardo Cunha de Almeida, and Felix Naumann. 2019. Discovery of Approximate (and Exact) Denial Constraints. Proc. VLDB Endow. 13, 3 (2019), 266-278.

[41] Meikel Pöss and Chris Floyd. 2000. New TPC Benchmarks for Decision Support and Web Commerce. SIGMOD Rec. 29, 4 (2000), 64-71.

[42] Tilmann Rabl, Manuel Danisch, Michael Frank, Sebastian Schindler, and HansArno Jacobsen. 2015. Just can't get enough: Synthesizing Big Data. In SIGMOD. 1457-1462.

[43] Theodoros Rekatsinas, Xu Chu, Ihab F. Ilyas, and Christopher Ré. 2017. HoloClean: Holistic Data Repairs with Probabilistic Inference. PVLDB 10, 11 (2017), 11901201.

[44] Anupam Sanghi, Raghav Sood, Jayant R. Haritsa, and Srikanta Tirthapura. 2018. Scalable and Dynamic Regeneration of Big Data Volumes. In EDBT. 301-312.

[45] William Sexton, John M. Abowd, Ian M. Schmutte, and Lars Vilhuber. 2017. Synthetic population housing and person records for the United States. https: //www.openicpsr.org/openicpsr/project/100274/version/V1/view

[46] Entong Shen and Lyublena Antova. 2013. Reversing statistics for scalable test databases generation. In DBTest. 7:1-7:6.

[47] Joshua Snoke and Aleksandra B. Slavkovic. 2018. pMSE Mechanism: Differentially Private Synthetic Data with Maximal Distributional Similarity. In PSD, Vol. 11126. 138-159.

[48] Ghanem Soltana, Mehrdad Sabetzadeh, and Lionel C. Briand. 2017. Synthetic data generation for statistical testing. In ASE. 872-882.

[49] Ghanem Soltana, Nicolas Sannier, Mehrdad Sabetzadeh, and Lionel C. Briand. 2018. Model-based simulation of legal policies: framework, tool support, and validation. Software and Systems Modeling 17, 3 (2018), 851-883.

[50] Latanya Sweeney. 2002. k-Anonymity: A Model for Protecting Privacy. Int. F. Uncertain. Fuzziness Knowl. Based Syst. 10, 5 (2002), 557-570.

[51] Y. C. Tay, Bing Tian Dai, Daniel T. Wang, Eldora Y. Sun, Yong Lin, and Yuting Lin. 2013. UpSizeR: Synthetically scaling an empirical relational database. Inf. Syst. 38, 8 (2013), 1168-1183.

[52] TPC. 2020. TPC-H benchmark. http://www.tpc.org/tpch/.

[53] Stanley Gill Williamson. 2002. Combinatorics for computer science. Courier Corporation.

[54] William E. Winkler. 2004. Masking and Re-identification Methods for Public-Use Microdata: Overview and Research Problems. In PSD, Vol. 3050. 231-246.

[55] Ran Yahalom, Erez Shmueli, and Tomer Zrihen. 2010. Constrained Anonymization of Production Data: A Constraint Satisfaction Problem Approach. In Secure Data Management, 7th VLDB Workshop, Vol. 6358. 41-53.

[56] Jun Zhang, Graham Cormode, Cecilia M. Procopiuc, Divesh Srivastava, and Xiaokui Xiao. 2017. PrivBayes: Private Data Release via Bayesian Networks. ACM Trans. Database Syst. 42, 4 (2017), 25:1-25:41. 